\title{
Receptor tyrosine kinases modulate distinct transcriptional programs by differential usage of intracellular pathways
}

\author{
Harish N Vasudevan, Pierre Mazot, Fenglei He, Philippe Soriano*
}

Department of Developmental and Regenerative Biology, Icahn School of Medicine at Mount Sinai, New York, United States

\begin{abstract}
Receptor tyrosine kinases (RTKs) signal through shared intracellular pathways yet mediate distinct outcomes across many cell types. To investigate the mechanisms underlying RTK specificity in craniofacial development, we performed RNA-seq to delineate the transcriptional response to platelet-derived growth factor (PDGF) and fibroblast growth factor (FGF) signaling in mouse embryonic palatal mesenchyme cells. While the early gene expression profile induced by both growth factors is qualitatively similar, the late response is divergent. Comparing the effect of MEK (Mitogen/Extracellular signal-regulated kinase) and PI3K (phosphoinositide-3-kinase) inhibition, we find the FGF response is MEK dependent, while the PDGF response is PI3K dependent. Furthermore, FGF promotes proliferation but PDGF favors differentiation. Finally, we demonstrate overlapping domains of PDGF-PI3K signaling and osteoblast differentiation in the palate and increased osteogenesis in FGF mutants, indicating this differentiation circuit is conserved in vivo. Our results identify distinct responses to PDGF and FGF and provide insight into the mechanisms encoding RTK specificity.
\end{abstract}

DOI: 10.7554/eLife.07186.001

*For correspondence: philippe. soriano@mssm.edu

Competing interests: The authors declare that no competing interests exist.

Funding: See page 18

Received: 25 February 2015

Accepted: 06 May 2015

Published: 07 May 2015

Reviewing editor: Marianne E Bronner, California Institute of Technology, United States

cc) Copyright Vasudevan et al. This article is distributed under the terms of the Creative Commons Attribution License, which permits unrestricted use and redistribution provided that the original author and source are credited.

\section{Introduction}

Receptor tyrosine kinases (RTKs) signal through a shared set of intracellular pathways, including extracellular signal-related kinase (ERK) and phosphatidylinositol 3-kinase (PI3K), yet the in vivo functions directed by different RTKs can be quite distinct, raising the question of how specific cellular responses are elicited (Lemmon and Schlessinger, 2010). Several models have been put forth to explain how RTKs encode specificity (Hunter, 2000; Simon, 2000; Pawson, 2004; Volinsky and Kholodenko, 2013). In concept, distinct responses may be encoded by modulation of individual pathways downstream of receptor activation, with each pathway regulating a specific outcome. Alternatively, many cellular responses may require integration from multiple input pathways, and signal specificity could arise from this unique combination of pathways. Analysis of mice harboring point mutations to disrupt binding of specific effector proteins to RTKs suggests both these models may apply in vivo; platelet-derived growth factor (PDGF) Receptor $\alpha$ (Pdgfra) mutants display effectorspecific phenotypes in line with the former model (Klinghoffer et al., 2002), but PDGF Receptor $\beta$ (Pdgfrb) mediated outcomes require combined output across multiple pathways, consistent with the latter model (Tallquist et al., 2003). In addition, quantitative differences in the duration and magnitude of signal induction provide an added layer of regulatory complexity (Marshall, 1995). At the transcriptional level, one outcome of RTK activation is the expression of immediate early genes (IEGs) (Cochran et alı, 1983; Lau and Nathans, 1987). Studies in cell culture have suggested IEGs constitute a generic readout of RTK activation with minimal specificity at the receptor or intracellular effector level (Fambrough et al., 1999), but genetic experiments in mice indicate a degree of IEG specificity (Schmahl et al., 2007). Therefore, a central goal remains to delineate RTK responsive 
eLife digest Cells produce many different proteins that play a variety of important roles. For example, proteins called receptor tyrosine kinases can detect particular molecules and send signals to other parts of the cell to regulate the activity (or "expression") of genes involved in cell division, movement, and other processes.

Humans have 58 receptor tyrosine kinases, and defects in these proteins have been linked to diseases such as cancer and diabetes. However, many different receptors regulate the activities of shared sets of genes, so it is not clear how an individual receptor can specifically control the genes involved in a particular process.

Two receptor tyrosine kinases called PDGFR and FGFR are crucial for the development of the face, palate, and head in humans and other animals. Vasudevan et al. used a technique called RNAsequencing to find out which genes are regulated by these receptors in mouse palate cells. The experiments show that there is a common set of genes whose activities change quickly-within 1 hour-in response to the activation of either PDGFR or FGFR. However, several hours later, cells in which PDGFR is activated have different patterns of gene expression compared to those with active FGFR.

Vasudevan et al. also found that FGFR promotes cell division, while PDGFR promotes the changing of palate cells into different types with more specialized roles. These different outcomes arise because PDGFR and FGFR use different signaling pathways that involve distinct proteins. For example, a protein called PI3K is critical for changes in gene expression in response to PDGFR but not FGFR.

These results suggest that PGDRF and FGFR control different cellular processes in the palate by sending distinct signals into the cell. Understanding the receptor tyrosine kinases and the networks of genes they activate will help us to identify the signals that are important for other processes, such as the development of the face.

DOI: 10.7554/eLife.07186.002

transcriptional programs, identify the key signaling parameters encoding their regulation, and determine how these gene expression profiles dictate cellular decisions.

Many components of RTK signaling play important roles in mammalian craniofacial development (Bentires-Alj et alo, 2006; Newbern et al., 2008; Fantauzzo and Soriano, 2015). In particular, PDGF and FGF signaling are both essential for midface development. In mice, loss of Pdgfra (Soriano, 1997) or its ligands Pdgfa and Pdgfc (Ding et al., 2004) results in facial clefting, and mice harboring a mutation abrogating PI3K binding to PDGFR $\alpha$ mirror these craniofacial phenotypes, implicating PI3K as the main effector of PDGFR $\alpha$ signaling (Klinghoffer et al., 2002). In addition, both Pdgfra (Wnt1Cre; Pdgfra ${ }^{f / f l}$ ) (Tallquist and Soriano, 2003) and FGF receptor 1 (Wnt1-Cre; Fgfr1) (Trokovic et alo, 2003; Wang et al., 2013) neural crest conditional mutants exhibit cleft face, indicating both pathways are required for normal development of the neural crest derived facial skeleton. At the intracellular pathway level, previous work has implicated ERK as a key effector downstream of FGF signaling (Lanner and Rossant, 2010). Furthermore, mutations in both PDGF and FGF signaling have been linked to craniofacial syndromes in humans (Choi et al., 2009; Miraoui and Marie, 2010; Rattanasopha et alo, 2012). Interestingly, chimeric receptor experiments in mice have shown that the intracellular domain of Fgfr1 cannot compensate for Pdgfra during development, suggesting these two receptors transmit biologically distinct signals in vivo (Hamilton et al., 2003). The midface thus offers a unique opportunity to interrogate the mechanisms of signal specificity between these two RTKs in a developmentally relevant system.

Given the requirement for PDGF and FGF signaling in the development of the neural crest derived midface, we sought to compare the gene expression programs regulated by these two RTKs. The architecture of the transcriptional response to RTK activation consists of three stereotypic waves: an IEG response involving core transcriptional regulators (Fos, Jun, Egr), a delayed response playing a feedback role (phosphatases, RNA-binding proteins), and a late sustained response determining cellular outcome (Amit et alo, 2007; Avraham and Yarden, 2011). However, the degree of conservation between genes regulated in each wave across different RTK families is unclear. Furthermore, although classic feedback regulators of mitogen-activated protein kinase (MAPK) 
pathways have been described, such as dual-specificity phosphatases (DUSPs) for ERK and c-Jun Nterminal kinase (JNK) (Li et al., 2007; Owens and Keyse, 2007), the extent of effector-dependent transcription genome-wide is not well characterized.

In the present work, we compare the transcriptional response to PDGF and FGF signaling in E13.5 mouse embryonic palatal mesenchyme (MEPM) cells. Although both PDGF and FGF are required in the neural crest for craniofacial development, we find distinct transcriptional programs, effector dependencies, and cellular outcomes in response to each RTK. While many genes in the early wave are shared across the two RTKs, FGF induces a quantitatively stronger response than PDGF. In addition, the feedback control provided by the delayed transcriptional wave displays distinct characteristics in response to PDGF and FGF. By exploring the effect of MEK/ERK and PI3K inhibition on these RTK-regulated gene expression profiles, we find PDGF-mediated transcription displays greater PI3K dependence, while FGF-mediated gene expression programs predominantly require ERK activity. This relationship is conserved at the level of cellular outcome, with FGF driving proliferation but PDGF promoting PI3K-dependent differentiation. Finally, we show overlapping domains of PI3K signaling, PDGF target gene expression, and skeletal differentiation during palatogenesis in vivo, a process perturbed in Fgfr1 conditional mutants. Taken together, our studies suggest unique roles for PDGF and FGF during development of the facial skeleton, and more broadly, demonstrate that distinct transcriptional responses to RTK signaling are encoded through qualitative and quantitative differences in intracellular pathway activation.

\section{Results}

\section{PDGF and FGF have distinct patterns of effector activation and transcriptional responses in E13.5 MEPMs}

Since neural crest conditional loss of either Fgfr1 or Pdgfra leads to clefting, we chose to perform RNA-seq on E13.5 MEPMs treated with either PDGFA or FGF1 + heparin to identify the gene expression programs regulated by each signaling pathway (Figure 1A). MEPMs express many essential markers of the palatal mesenchyme and have been previously used to study responses to many pathways (Bush and Soriano, 2010; Iwata et al., 2012; Fantauzzo and Soriano, 2014), including PDGF and FGF (Vasudevan and Soriano, 2014). We performed RNA-seq at 1 and $4 \mathrm{hr}$ following ligand treatment in order to characterize both the early and late responses to PDGF and FGF signaling (Supplementary File 1). In the samples submitted for sequencing, both PDGF and FGF induced a robust phospho-ERK ( $p E R K$ ) response at $15 \mathrm{~min}$ (Figure 1-figure supplement 1A), and MEPMs generated from Pdgfra-GFP (Hamilton et al., 2003) and Fgfr1-CFP (to be described elsewhere) knockin reporter embryos display expression of each receptor at the protein level in all cells (Figure 1-figure supplement 1B), further validating MEPMs as a suitable system to study RTK responses.

We first plotted the expression of all genes with FPKM (fragments per kilobase of exon per million reads mapped) values $>1$ at both $1 \mathrm{hr}$ (Figures $1 \mathrm{~B}$ and $4 \mathrm{hr}$ (Figure 1B'); although only a small number of genes are differentially regulated between the 1-hr PDGF and 1-hr FGF samples (Cuffdiff $\mathrm{q}<0.1$, Supplementary File 2; Trapnell et alo, 2010), the difference in the response to these two growth factors is much greater at $4 \mathrm{hr}$. Consistent with this observation, visualization of all replicates by principal component analysis (PCA) (Figure 1-figure supplement 1C) revealed that the 1-hr PDGF and 1-hr FGF samples cluster together, but the 4-hr FGF replicates are distinctly separate from the 4-hr PDGF samples. Comparing the stimulated MEPMs to untreated cells, genes differentially regulated at $1 \mathrm{hr}$ by either PDGF or FGF (Supplementary File 2) show high correlation $\left(r^{2}=0.8173\right.$, Figure $1 C)$, but by $4 \mathrm{hr}$, the two RTK signals are divergent $\left(r^{2}=0.2881\right.$, Figure $\left.1 \mathrm{C}^{\prime}\right)$. In addition, the genes regulated by PDGF at $1 \mathrm{hr}(n=40)$ form a subset of those genes regulated by FGF at $1 \mathrm{hr}(n=$ 159), further highlighting the similarity within the early response to both growth factors. Gene ontology analysis (Huang et al., 2009) of the genes induced at $1 \mathrm{hr}$ revealed an enrichment of transcription factors and MAP kinase phosphatases downstream of both RTKs (Figure 1-figure supplement 1D, $\mathrm{p}<0.001$ ), similar to previous descriptions of the response to RTK activation (Amit et al., 2007; Avraham and Yarden, 2011). To better visualize the organization of these targets, we constructed a protein-protein interaction (PPI) network from the genes regulated at $1 \mathrm{hr}$; in constructing this network, we only included direct interactions between proteins (path length $=1$ ) and excluded predicted interactions (Berger et al., 2007; Chen et al., 2012). The resulting network 


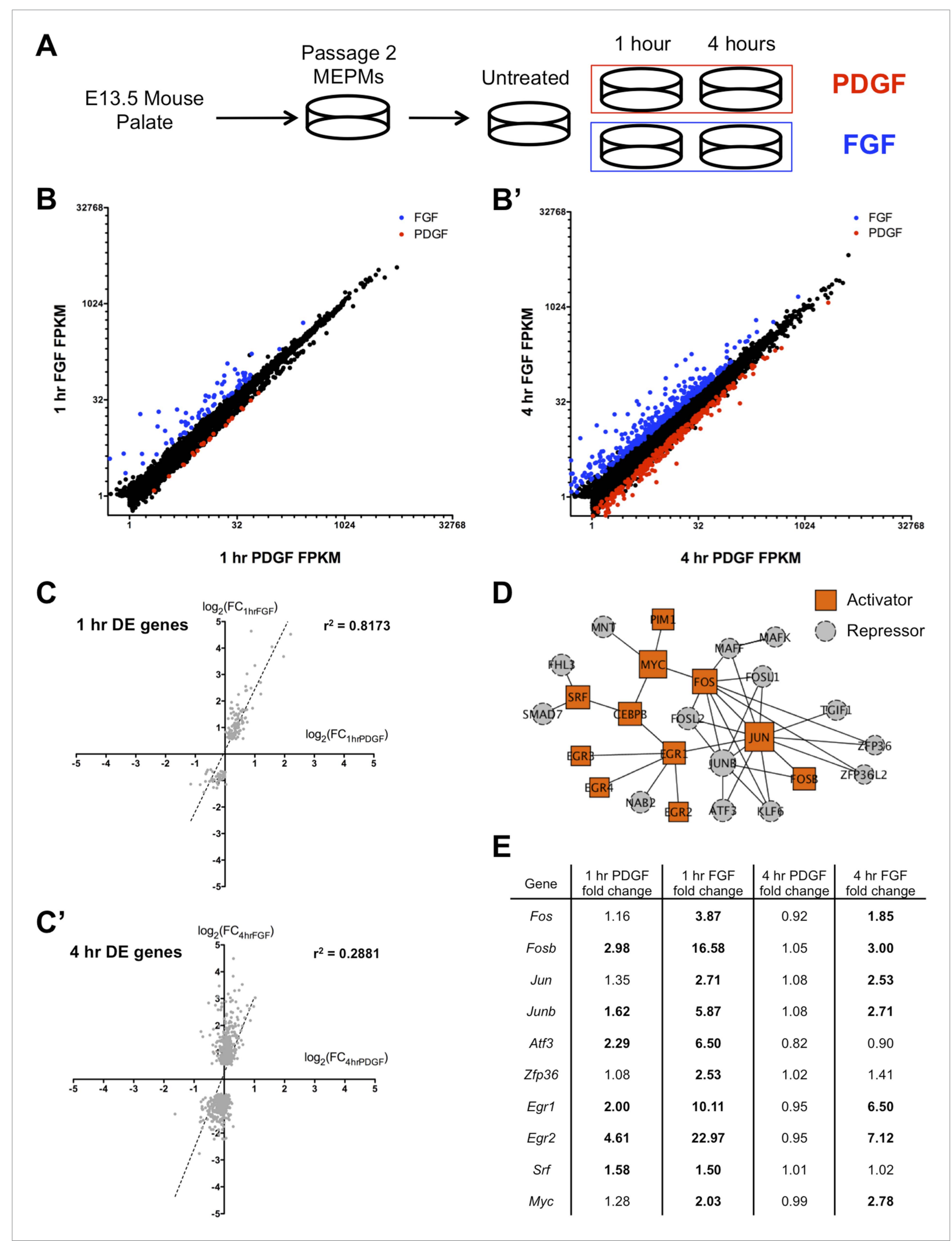

Figure 1. FGF and PDGF stimulation result in distinct transcriptional responses. (A) Mouse embryonic palatal mesenchyme (MEPM) cells were dissected from E13.5 embryos, passaged twice, and then serum starved overnight prior to stimulation with either PDGFAA or FGF1 + heparin. (B) Expression of all genes with FPKM (fragments per kilobase of exon per million reads mapped) $>1$ at $1 \mathrm{hr}(11,217$ genes) and (B') 4 hr (11,266 genes). Genes colored blue are Figure 1. continued on next page 
Figure 1. Continued

significantly increased with fibroblast growth factor (FGF) treatment and genes colored red are significantly increased with platelet-derived growth factor (PDGF) treatment. Values plotted on $\log _{2}$ scale. (C) Fold change (FC) comparison for all differentially expressed (DE) genes at (C) $1 \mathrm{hr}$ or (C') 4 hr

(compared to serum starved sample) shows high correlation between the transcriptional response to each growth factor at $1 \mathrm{hr}$ but low correlation at $4 \mathrm{hr}$. (D) Protein-protein interaction (PPI) network for all genes upregulated at $1 \mathrm{hr}$ contains many classic immediate early genes (such as AP-1 components, Myc, and Srf). Genes are colored based on their primary reported role in transcriptional regulation $(22,23)$, with orange squares representing activators and gray circles representing repressors. (E) FC (compared to untreated sample) for selected genes upregulated at 1 hr. Genes in bold are induced $>1.5$-fold in response to the indicated growth factor. Although both PDGF and FGF regulate many shared targets, the induction in response to FGF exhibits greater magnitude (Fos, Fosb, Junb, Atf3, Egr1, Egr2) and longer duration (Fos, Fosb, Jun, Junb, Egr1, Egr2).

DOI: 10.7554/eLife.07186.003

The following figure supplement is available for figure 1:

Figure supplement 1. Characterization of E13.5 MEPMs and transcriptional response to PDGF and FGF signaling. DOI: 10.7554/eLife.07186.004

contains 25 upregulated genes (out of 113 total induced genes), including many classic components of the IEG response, such as activator protein-1 (AP-1) subunits (Fos, Jun) and their regulators (Zfp36, Atf3) as well as Myc, Egr1-4, and Srf(Figure 1D). Closer inspection revealed that many shared target genes within this network are regulated to both a stronger magnitude and longer duration following FGF treatment compared to PDGF treatment (Figure 1E). Indeed, Fos, Fosb, Jun, and Junb all exhibit differences in signal magnitude and/or duration in response to FGF, as validated by qPCR (Figure 1-figure supplement 1E). In sum, the two RTKs induce a similar gene expression profile at $1 \mathrm{hr}$ (Figure 1C), as evidenced by the high-correlation coefficient and overlap between genes differentially regulated by PDGF and FGF at $1 \mathrm{hr}$. However, FGF drives a quantitatively stronger early response with many transcription factors showing both a stronger magnitude and greater duration of induction in response to FGF compared to PDGF (Figure 1E), which may explain in part the divergent gene expression profiles observed at $4 \mathrm{hr}$.

\section{Differential roles for the delayed transcriptional response in regulation of signaling downstream of FGF and PDGF}

Given the importance of MEK/ERK and PI3K/Akt signaling downstream of these RTKs during development (Klinghoffer et al., 2002; Corson et al., 2003; Lanner and Rossant, 2010; Fantauzzo and Soriano, 2014), we analyzed pERK and pAkt activation following FGF and PDGF stimulation in MEPMs (Figure 2A). Consistent with the stronger response to FGF treatment in the gene expression data, the FGF-induced pERK response displays both a higher magnitude and longer duration of activation compared to the PDGF-induced pERK signal. In contrast, both FGF and PDGF induce similar patterns of pAkt activation, but the magnitude of the PDGF-pAkt induction is slightly greater. The FGF-pERK signal is apparent up to $6 \mathrm{hr}$ following growth factor treatment (Figure 2-figure supplement 1A), and increasing the dose of PDGFA ligand did not alter the kinetics of pERK activation (Figure 2-figure supplement 1B). To better understand differences in the response to PDGF and FGF, we performed gene ontology analysis for the molecular function of genes that are differentially expressed (DE) between 4-hr PDGF treatment and 4-hr FGF treatment (Figure 2B, $p<0.001$ ) (Huang et al., 2009). The top results for genes enriched following FGF treatment are sets associated with modulation of signaling, such as protein kinases and GTPase regulators, which may function as activators of Ras to promote MEK/ERK signaling. Transcriptional feedback regulation of RTK signaling is well established, particularly the role of DUSPs providing negative feedback for MAPK signaling (Amit et al., 2007; Li et al., 2007; Owens and Keyse, 2007). Indeed, many DUSPs (MAPK phosphatases) are induced in response to both PDGF and FGF treatment at $1 \mathrm{hr}$ (Figure 1-figure supplement 1D), but FGF alone induces the expression of kinases and GTPase regulators at $4 \mathrm{hr}$, suggesting a distinct role for the FGF response in regulating MEK/ERK activity. We, thus, performed Western blots in the presence of cycloheximide following both PDGF and FGF treatment to determine the effect of inhibiting protein synthesis (and consequently, the delayed transcriptional response) on ERK activation. Consistent with previous work exploring the role of DUSPmediated negative feedback (Amit et al., 2007), cycloheximide treatment increased the duration of the PDGF-pERK response (Figure 2C). However, we observed the opposite effect of cycloheximide treatment on the FGF-pERK response (Figure 2C'), suggesting a positive feedforward loop in which 

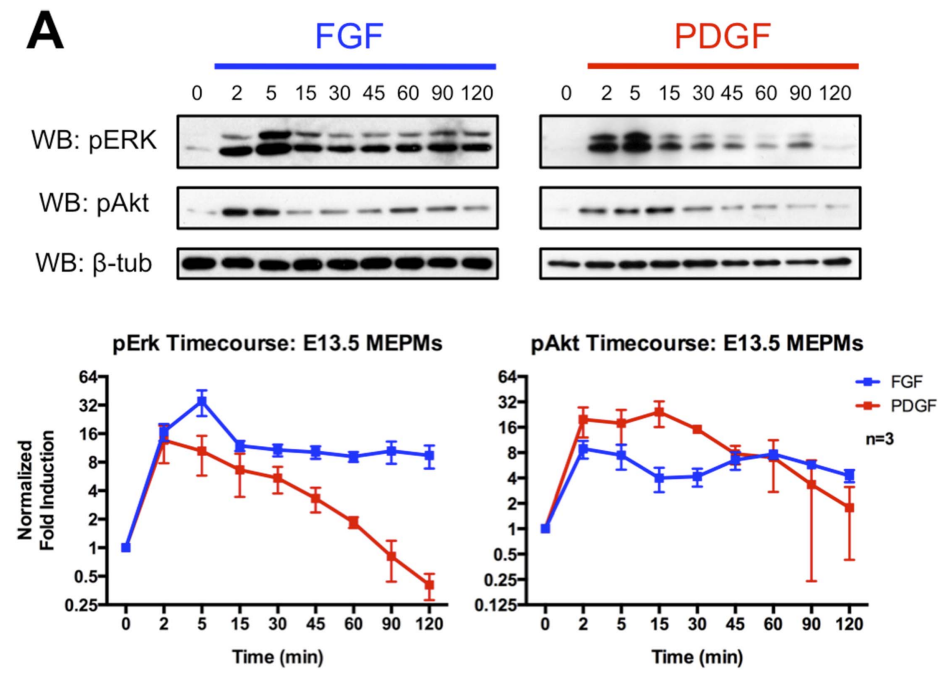

B

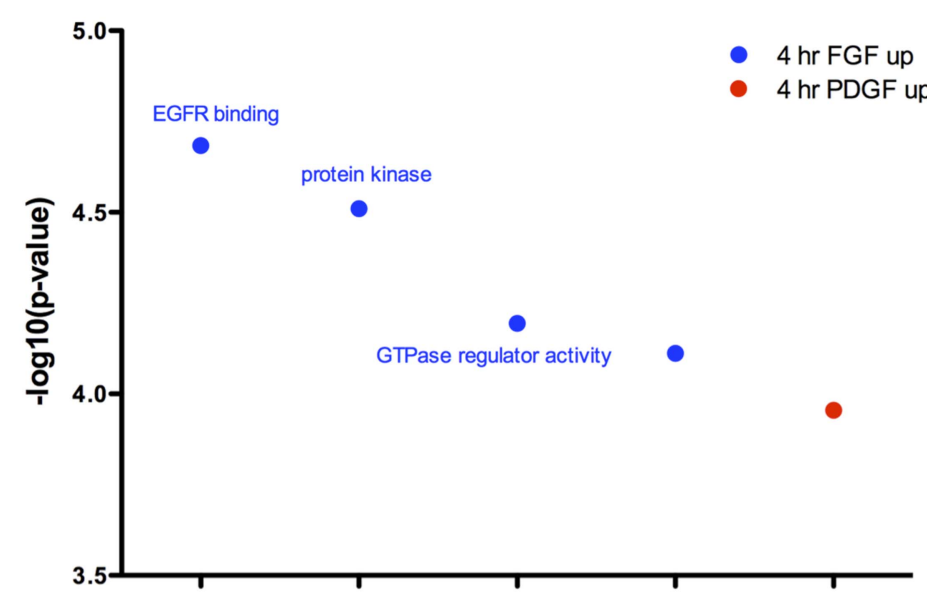

C PDGF (min)

- $1560120-1560120$

Chx - - - + + +

WB: pERK

WB: $\beta$-tub $-\ldots---$

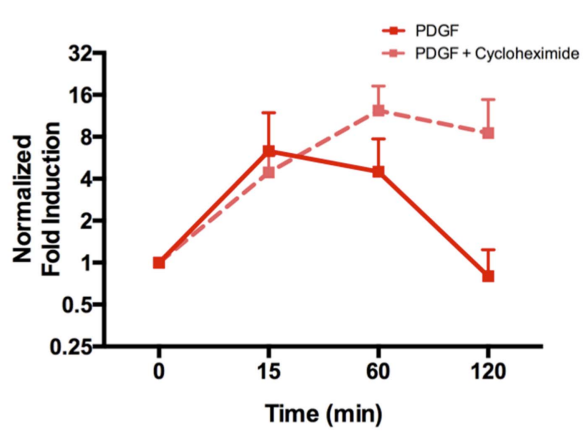

C'

FGF (min)

- 1560120 - 1560120

Chx - - - - + + + +

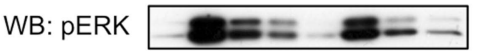

WB: $\beta$-tub

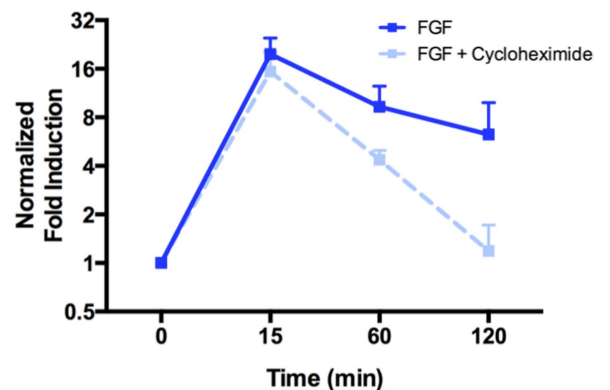

D

$\longrightarrow$ Transcriptional negative feedback loop

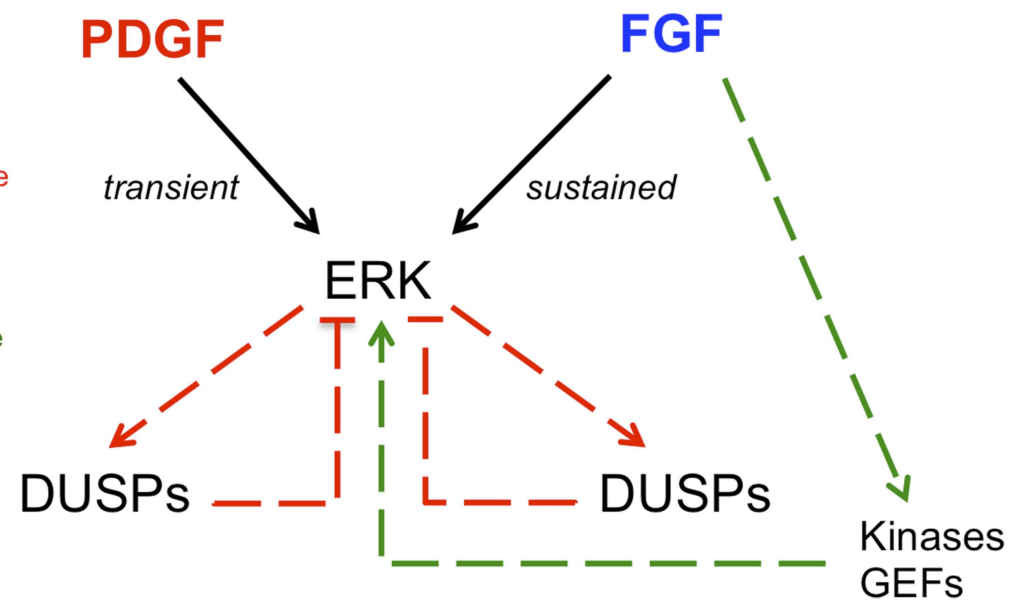

Figure 2. The delayed transcriptional response provides differential regulation of pERK duration in response to FGF and PDGF signaling. (A) Signaling time course shows a more robust phospho-ERK (pERK) response to FGF (blue) than PDGF (red) and a similar pAkt response to both growth factors.

(B) Gene ontology analysis (molecular function) of genes DE between the 4-hr PDGF and 4-hr FGF conditions indicates enrichment for kinases and GTPase Figure 2. continued on next page 
Figure 2. Continued

regulators in response to FGF signaling. (C) Cycloheximide treatment has opposite effects on pERK duration following (C) PDGF and (C') FGF stimulation, indicating the delayed transcriptional response (dependent on protein synthesis and thus inhibited by cycloheximide) can provide both negative and positive signals to modulate pERK kinetics. (D) Model depicting loops that regulate the duration of the pERK wave in response to receptor tyrosine kinase (RTK) signaling includes both negative (dual-specificity phosphatases [DUSPs]) and positive (kinases, GEFs) components from the delayed transcriptional response. Western blot quantification plotted as mean $\pm \mathrm{SEM}, \mathrm{n}=3$.

DOI: 10.7554/eLife.07186.005

The following figure supplement is available for figure 2:

Figure supplement 1. Signaling kinetics and organization of the late transcriptional response in response to PDGF and FGF treatment. DOI: 10.7554/eLife.07186.006

FGF induces the expression of kinases and GEFs to modulate the ERK response in addition to activating ERK directly. To further explore the architecture of the late FGF response, we constructed a PPI network from genes increased at 4-hr FGF stimulation compared to 4-hr PDGF treatment. The FGF network (Figure 2-figure supplement 1C) contains Prkca as a highly connected node, which is interesting given reported roles for protein kinase $C$ (PKC) in facilitating a sustained pERK response (Bhalla et al., 2002; Santos et al., 2007) as well as its importance downstream of FGF in skeletal development (Miraoui and Marie, 2010). We found that inhibition of PKC decreased both the initial pulse and sustained activation of the FGF-mediated pERK response in MEPMs (Figure 2-figure supplement 1C'), consistent with its potential function as a hub within the FGF response network. In addition, many of the kinases and GEFs transcriptionally regulated by FGF have reported roles in positively modifying MEK/ERK signaling (Figure 2-figure supplement 1D), which may explain in part the residual pERK response to FGF in the presence of PKC inhibition. Collectively, these data support a model in which the balance between positive and negative transcriptional loops is critical for determining patterns of pERK response to different RTKs (Figure 2D).

\section{The FGF transcriptional response is primarily MEK/ERK dependent, while the PDGF response shows greater PI3K usage}

The differences in signaling pathway activation following PDGF and FGF stimulation led us to consider how inhibition of these pathways affected the two RTK-mediated transcriptional programs. Thus, we analyzed the effector dependence of the transcriptional response by performing RNA-seq in cells stimulated with either PDGF or FGF in the presence of PD325901 (MEK inhibitor) or LY294002 (PI3K inhibitor) (Supplementary File 1). PCA on all thirteen sequenced conditions (Figure 3-figure supplement 1A) segregates the samples based on growth factor treatment along PC1 (44.57\% of the variance) and on inhibitor treatment along PC2 (17.72\% of the variance). Similarly, the correlation matrix for all sampled replicates mimics the PCA, with the $4 \mathrm{hr}$ FGF and $4 \mathrm{hr}$ FGF + LY showing a gene expression profile distinct from all other samples (Figure 3-figure supplement 1B). We next directly tested the effect of pathway inhibition on both the shared gene expression program induced by the two RTKs at $1 \mathrm{hr}$ (113 genes total) and the genes regulated between FGF and PDGF treatment at $4 \mathrm{hr}$. Globally, FGF target genes show greater MEK/ERK dependence than PI3K dependence, while PDGF responsive genes exhibit the opposite relationship (Supplementary File 3) mirroring the reported signaling requirements for each RTK. This trend is apparent at $1 \mathrm{hr}$ (Figure 3A, A) and striking for genes DE at $4 \mathrm{hr}$, where $52 \%$ of FGF responsive genes are MEK/ERK dependent (Figure $3 \mathrm{~B}$ ) but only $22 \%$ were PI3K dependent (Figure 3B'). This dependence is inverted for PDGF, where $9 \%$ are MEK/ERK dependent (Figure 3 C) but $28 \%$ are PI3K dependent (Figure 3C'). Strikingly, MEK inhibition increases the expression of PDGF targets (Figure 3C) but not FGF targets (Figure 3B), while PI3K inhibition increases the expression of FGF responsive genes (Figure $3 A^{\prime}$, B'), suggesting the FGF-ERK and PDGF$\mathrm{PI} 3 \mathrm{~K}$ relationships are important for both gene induction and repression. These intracellular pathway dependencies are independent of the magnitude of induction/repression, as varying the FC threshold did not affect the PDGF-PI3K or FGF-ERK relationships (Figure 3-figure supplement 1C, D').

We then considered the degree of overlap between ERK and PI3K targets. At $1 \mathrm{hr}$, many shared target genes show similar ERK or PI3K dependence, possibly constituting a core pathway specific gene set (Figure 3D). On the other hand, cross-comparison of PI3K- and ERK-dependent genes reveals some overlap (Figure 3D'), underscoring a degree of plasticity in pathway usage downstream 
A 1 hour upregulated + PD

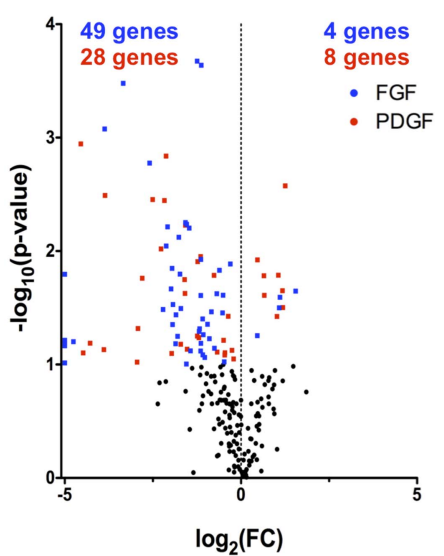

B

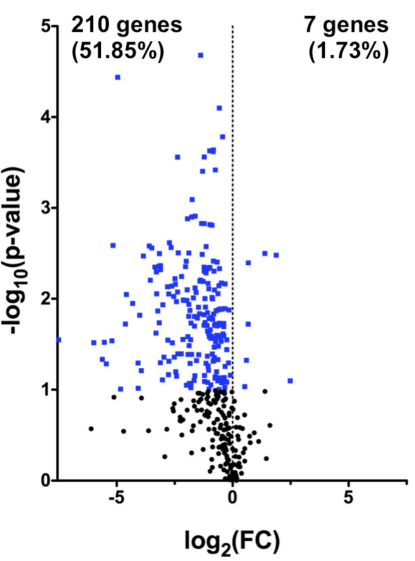

C 4 hour PDGF+PD

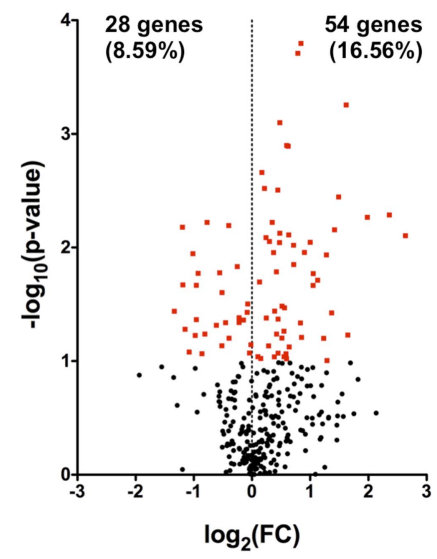

A' 1 hour upregulated $+\mathrm{LY}$

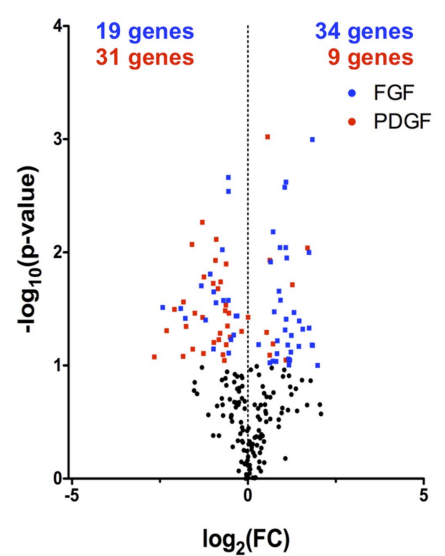

D
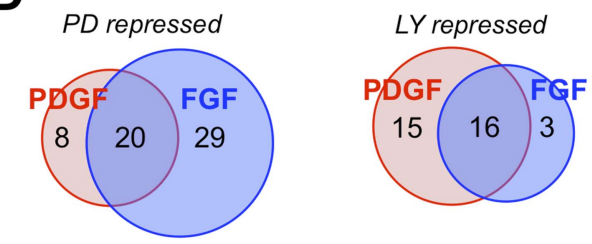

D'

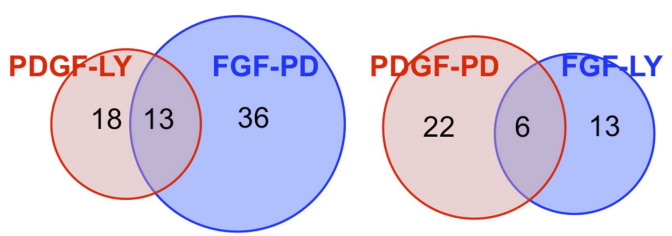

E

4 hour DE genes
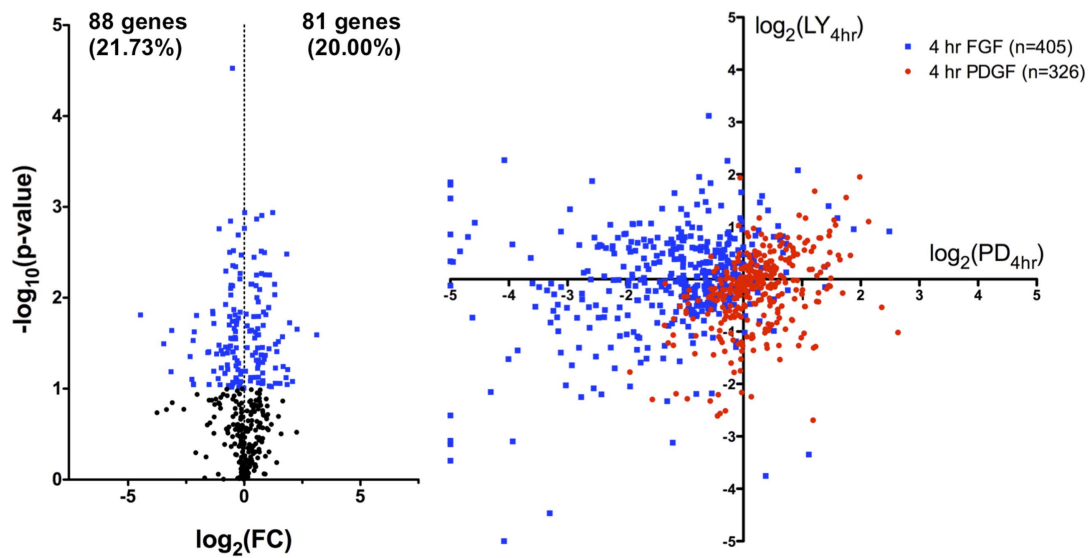

$E^{\prime}$

C' 4 hour PDGF+LY

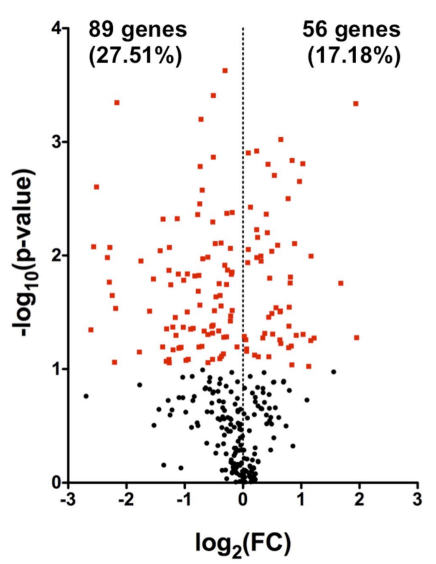

4 hour DE genes

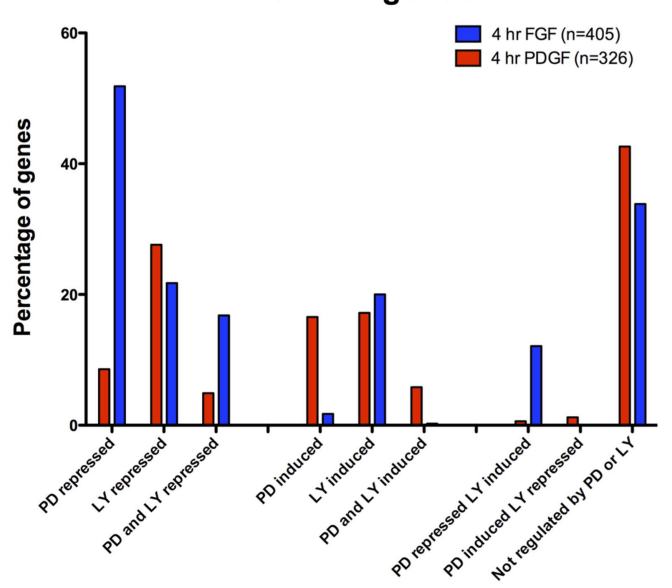

Figure 3. FGF and PDGF transcriptional responses exhibit differential usage of intracellular pathways. (A) Volcano plots visualizing the effect of (A) MEK (Mitogen/Extracellular signal-regulated kinase) inhibition and ( $\left.\mathbf{A}^{\prime}\right)$ phosphatidylinositol 3-kinase (PI3K) inhibition on the shared set of 113 genes upregulated at $1 \mathrm{hr}$ reveal that FGF (blue points) shows increased dependence on MEK/extracellular signal-related kinase (ERK) signaling, while PDGF Figure 3. continued on next page 
Figure 3. Continued

(red points) utilizes PI3K to a greater degree. X-axis plotted as $\log _{2}([1 \mathrm{hr}$ ligand + inhibitor]/[1 hr ligand]). (B) Genes with increased expression at 4-hr FGF treatment show higher dependence on (B) MEK/ERK activity compared to (B') PI3K. (C) In contrast, genes with increased expression at 4-hr PDGF treatment show greater usage of (C') PI3K compared to (C) MEK/ERK. X-axis plotted as $\log _{2}([4 \mathrm{hr}$ ligand + inhibitor $] /[4 \mathrm{hr}$ ligand]). Data analyzed using two sample t-test, and genes at $p<0.1$ are colored significant. Black points represent genes not significant at this threshold in all plots. (D) A core set of MEK/ ERK (20 genes) and PI3K (16 genes) are dependent on these pathways downstream of both PDGF and FGF. (D') A minority of genes can be activated through either MEK/ERK or PI3K signaling in response to PDGF or FGF, indicating a degree of plasticity in intracellular pathway usage. (E) Scatter plot comparing effect of MEK/ERK and PI3K inhibition on all $4 \mathrm{hr}$ DE genes reflects FGF-ERK and PDGF-PI3K dependencies. Data plotted as log 2 ([4 hr ligand + inhibitor]/[4 hr ligand]) and capped at \pm 5 for visualization. (E') 52\% of FGF target genes at $4 \mathrm{hr}$ are repressed by MEK/ERK inhibition, while $28 \%$ of PDGF responsive genes are repressed by PI3K inhibition. Interestingly, 12\% of genes are repressed by MEK/ERK inhibition and 'superinduced' by PI3K inhibition, indicating crosstalk between these pathways. Furthermore, 43\% of PDGF responsive genes and 34\% of FGF responsive genes are not significantly affected by either inhibitor, which suggests either combinatorial requirement of MEK and PI3K or alternate intracellular pathways drive expression of these genes. DOI: 10.7554/eLife.07186.007

The following figure supplement is available for figure 3 :

Figure supplement 1. RTK target genes show distinct patterns of effector dependence in a threshold-independent manner. DOI: 10.7554/eLife.07186.008

of different RTKs. When directly comparing the effect of MEK and PI3K inhibition at 4 hr (Figure 3E), the crosstalk between FGF targets is particularly striking, as a large number of genes repressed by MEK inhibition were regulated (both positively and negatively) by PI3K inhibition. Furthermore, while $23 \%$ of PDGF targets are specifically PI3K dependent, only $5 \%$ of FGF targets are repressed by PI3K alone (Figure $3 E^{\prime}$ ). Instead, many FGF-PI3K targets are also repressed by MEK inhibition (77\%), and conversely, a number of FGF-ERK targets are induced by PI3K inhibition (23\%), indicating the effect of PI3K inhibition downstream of FGF involves crosstalk with ERK signaling.

\section{Inhibition of intracellular effector activation leads to induction of alternate signaling pathways}

We were intrigued by the subset of genes exhibiting significantly increased expression ('superinduction') following inhibitor treatment at both 1 and $4 \mathrm{hr}$. Both RTKs show increased Jun expression in response to PD325901, while FGF and LY294002 treatment upregulates many classic ERK targets such as Fos, Fosb, and Dusp6 (Supplementary File 3), suggesting this 'superinduction' may reflect compensatory activation of other intracellular pathways. Many such examples of crosstalk between ERK and PI3K have been documented (Mendoza et al., 2011). Thus, we assayed the activation of these pathways following MEK inhibition with PD325901 and PI3K inhibition with LY294002. We found striking induction of pJNK upon pERK inhibition by PD325901 downstream of both FGF (Figure 4A) and PDGF (Figure 4B) stimulation. Interestingly, a moderate change in pAkt induction following PDGF treatment and MEK inhibition seems apparent, although this induction was not observed at the dose used for the RNA-seq experiment ( $1 \mu \mathrm{M}$ PD325901). Next, we investigated the effect of PI3K inhibition with LY294002. We found that FGF-mediated pERK induction increased with LY294002 treatment (Figure 4A), but PDGF treatment did not produce this increase in pERK signal (Figure 4B'), consistent with changes observed at the level of target gene expression. At the inhibitor doses used in the RNA-seq experiment (1 $\mu \mathrm{M}$ PD325901, $10 \mu \mathrm{M}$ LY294002), both PDGF and FGF significantly induce JNK activation in the presence of MEK inhibition, while only FGF activates ERK when PI3K is inhibited (Figure 4C, C'). Finally, we performed qPCR for a subset of target genes to confirm their response to pathway inhibition. Fos, Fosb, and Junb exhibit 'superinduction' downstream of FGF specifically in response to PI3K inhibition, confirming these genes are indeed MEK/ERK dependent and providing further evidence that LY294002-mediated induction of pERK can drive transcriptional changes (Figure 4D). Similarly, Jun is 'superinduced' in the presence of PD325901 downstream of both RTKs. There is a modest increase in Jun induction following FGF treatment with PI3K inhibition, likely reflecting the compensatory induction of pERK in this condition and consequent crosstalk between ERK and JNK signaling. In addition, Fos induction is increased with LY294002 treatment even in the absence of growth factor, which may reflect a degree of growth factor independent crosstalk between PI3K and ERK. However, this effect synergizes with ligand treatment, indicating this compensation across intracellular pathways is at least partially dependent on receptor activation. 


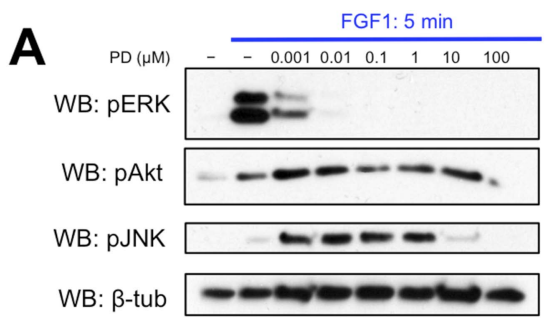

$A^{\prime}$

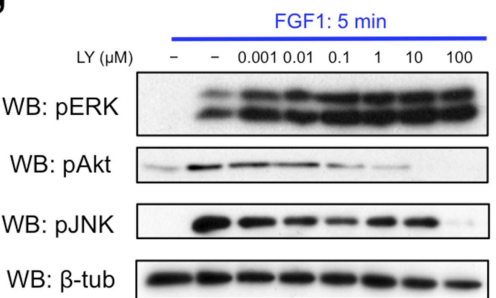

C

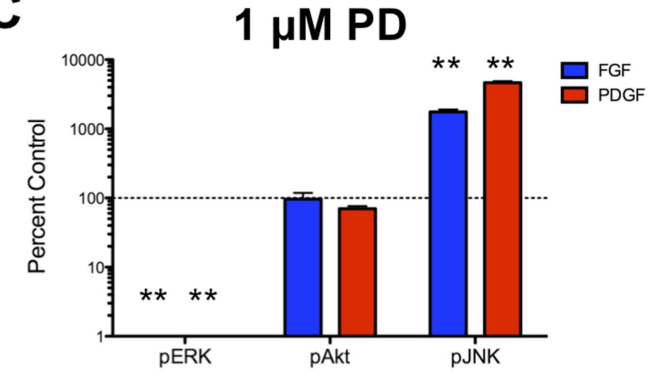

D
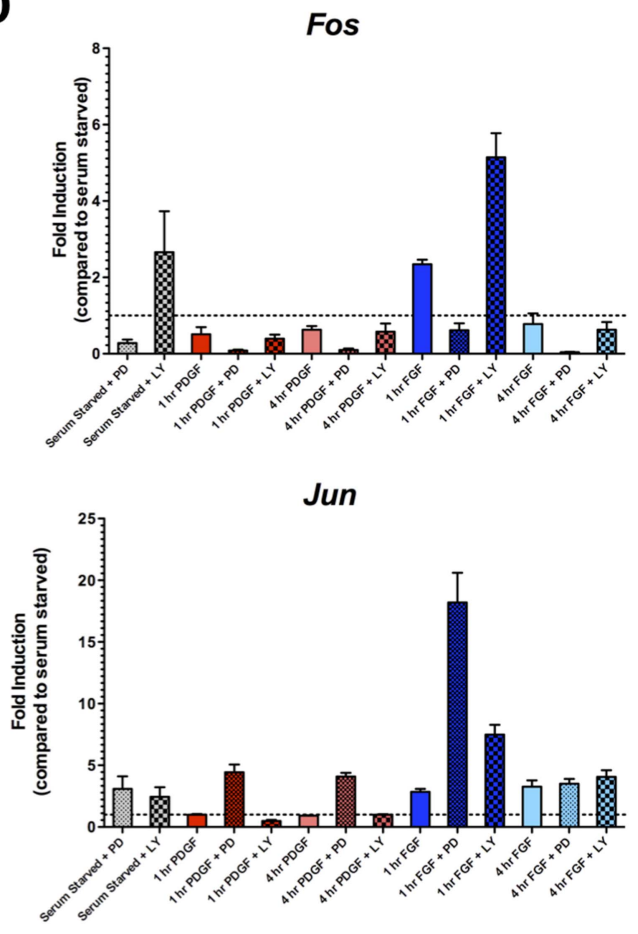

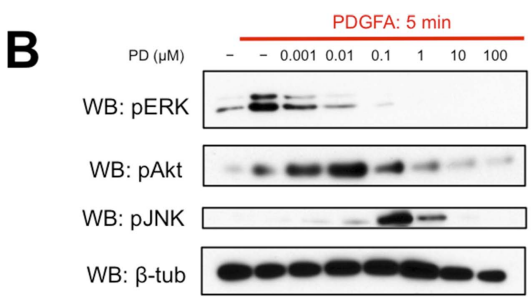

B'

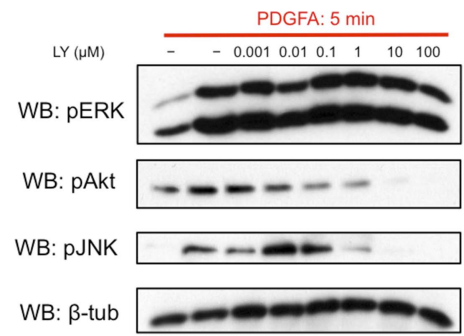

C'

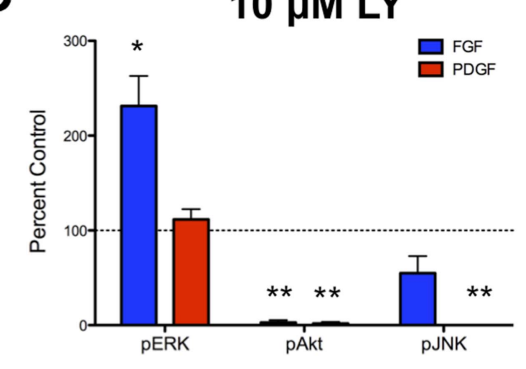

Fosb

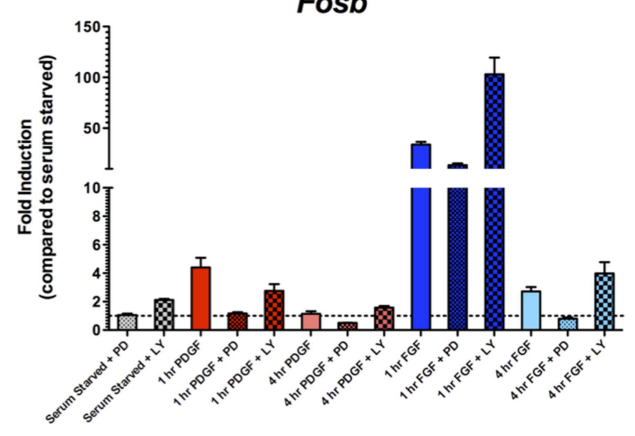

Junb

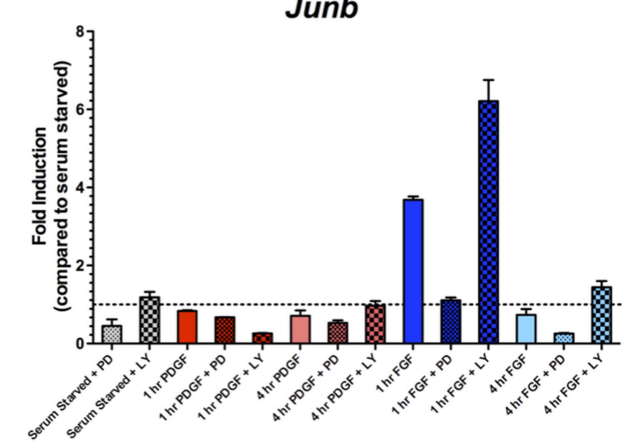

Figure 4. Inhibition of effector activation results in compensatory induction of alternate signaling pathways detectable in the transcriptional response. (A) PD325901 dose response Western blots reveal induction of pJNK as pERK is progressively inhibited downstream of FGF signaling. (A') Similarly, LY294002 dose response Western blots reveal increased pERK signal as pAkt is inhibited. (B) Inhibitor dose response Western blots in response to PDGF signaling show activation of c-Jun N-terminal kinase (JNK) in response to MEK/ERK inhibition but (B') no activation of ERK following PI3K inhibition. Figure 4. continued on next page 
Figure 4. Continued

(C) Quantification of effector activation in response to FGF (blue) or PDGF (red) at the doses used in the RNA-seq experiment reflects (C) increased pJNK activation when MEK/ERK signaling is inhibited and $\left(\mathbf{C}^{\prime}\right)$ increased pERK induction when PI3K activity is blocked. Data plotted as mean \pm SEM, $n=3$ and compared using two sample, unpaired t-test to baseline of $100 \%$ (no change). ${ }^{*} p<0.05 ;{ }^{* \star} p<0.001$. (D) Gene expression reflects the crosstalk observed at the signaling level, as verified by qPCR for selected target genes. Canonical ERK targets such as Fos, Fosb, and Junb are 'superinduced' upon LY treatment, while the JNK target Jun is 'superinduced' with PD treatment. Interestingly, a degree of 'superinduction' is observed in the presence of inhibitor prior to growth factor addition, which may reflect RTK-independent crosstalk between intracellular pathways. Data plotted as mean \pm SEM, $n=3$. DOI: 10.7554/eLife.07186.009

\section{FGF drives MEK/ERK-dependent cell proliferation, and PDGF promotes PI3K-dependent cell differentiation}

We next investigated the functional consequence of these differential effector activation patterns and transcriptional programs in response to PDGF and FGF signaling. Gene ontology for biological processes (Huang et al., 2009) enriched in genes differentially regulated by PDGF and FGF at $4 \mathrm{hr}$ revealed an FGF-mediated proliferation program and a PDGF-regulated differentiation circuit (Figure 5A, $\mathrm{p}<0.001$ ). Interestingly, we also observed enrichment for regulators of Wnt signaling in the 4-hr PDGF condition, consistent with reports of antagonism between Wnt and FGF as regulating the balance between differentiation and proliferation in skeletal development (Mansukhani et al., 2005). We further identified a set of differentiation genes (Id1, Id2, Id3, Mef2c, Atoh8) in the PPI network constructed from genes regulated by PDGF treatment at $4 \mathrm{hr}$ (Figure 5-figure supplement 1A) and confirmed these genes by qPCR (Figure 5-figure supplement 1A). In line with PDGF directing a skeletal differentiation program, the Id genes (Maeda et al., 2004; Kee and Bronner-Fraser, 2005) and Mef2c (Verzi et al., 2007) are required for craniofacial skeleton development in vivo. In addition, mouse genome informatics mammalian phenotype analysis to determine overrepresented mouse phenotypes (Chen et al., 2013) identifies abnormal craniofacial bone development as the most enriched phenotype in the 4-hr PDGF target genes (Figure 5-figure supplement 1B, B). Plotting the expression of these FGF-proliferation and PDGF-differentiation genes emphasizes the distinct responses of these gene sets to these two growth factors (Figure 5B). Furthermore, many proliferation genes are reduced upon MEK inhibition and induced upon PI3K inhibition, while the opposite is apparent for differentiation genes, consistent with the FGF-ERK and PDGF-PI3K dependencies observed globally at the transcriptional level.

To directly assay cell proliferation, we performed BrdU labeling at $4 \mathrm{hr}$ following either FGF or PDGF treatment in E13.5 MEPMs; while PDGF induces a modest response compared to serumstarved cells, we found a significantly greater proliferative response to FGF (Figure $5 \mathrm{C}$ ). Crystal violet staining for cell viability confirms a greater effect of FGF than PDGF as well as the importance of MEK/ ERK activity for this response (Figure 5-figure supplement 1C). Furthermore, the PDGF-dependent effect on cell viability is significantly greater at day 3 compared to the $0.1 \%$ fetal bovine serum (FBS) treated cells, underscoring the role of PDGF in cell survival/growth in the absence of other growth factors. We next measured apoptosis following PDGF and FGF treatment in the presence of both inhibitors (Figure 5-figure supplement 1D). Consistent with the cell viability results and phosphoJNK induction patterns, MEK inhibition following FGF stimulation results in a greater increase in apoptosis than inhibition of FGF-mediated PI3K signaling; in contrast, PI3K inhibition has a greater effect than MEK inhibition downstream of PDGF stimulation, although inhibiting either pathway results in increased apoptosis. Finally, we tested cell differentiation by alkaline phosphatase (AP) staining, a marker of osteoblast differentiation ( $W u$ et al., 2008). PDGF-treated MEPMs display a robust AP response, while FGF-treated cells show a striking lack of AP positive cells (Figure 5D). In addition, MEK/ERK inhibition increases AP staining, but PI3K inhibition represses osteoblast differentiation (Figure 5D). These experiments suggest the following model: FGF drives cell proliferation and represses cell differentiation in a MEK/ERK-dependent manner, while PDGF facilitates cell differentiation, at the expense of reduced proliferation, in a PI3K-dependent manner.

\section{The FGF repressed, PI3K-dependent differentiation circuit is conserved during mouse craniofacial development}

Finally, we sought to investigate the FGF-ERK-proliferation and PDGF-PI3K-differentiation axes in vivo. We first examined the expression pattern of Fgfr1 in relation to Dusp6 (ERK-specific), Dusp1 
A

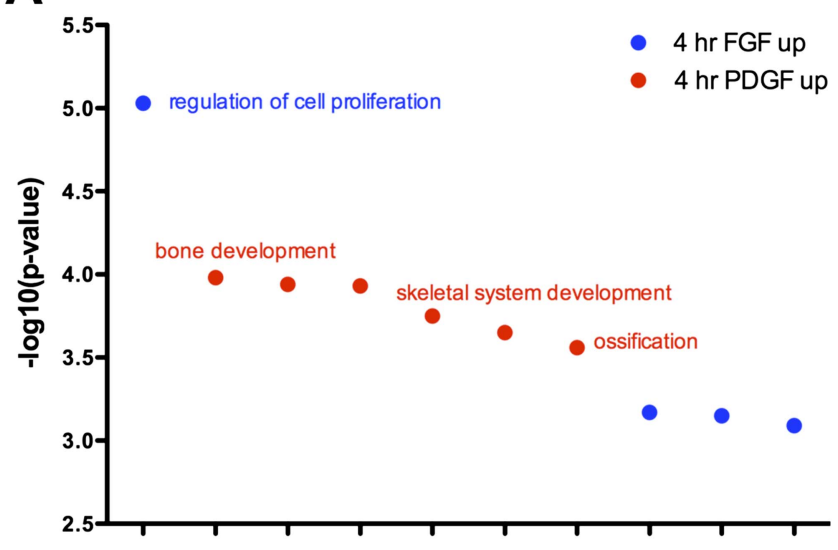

C
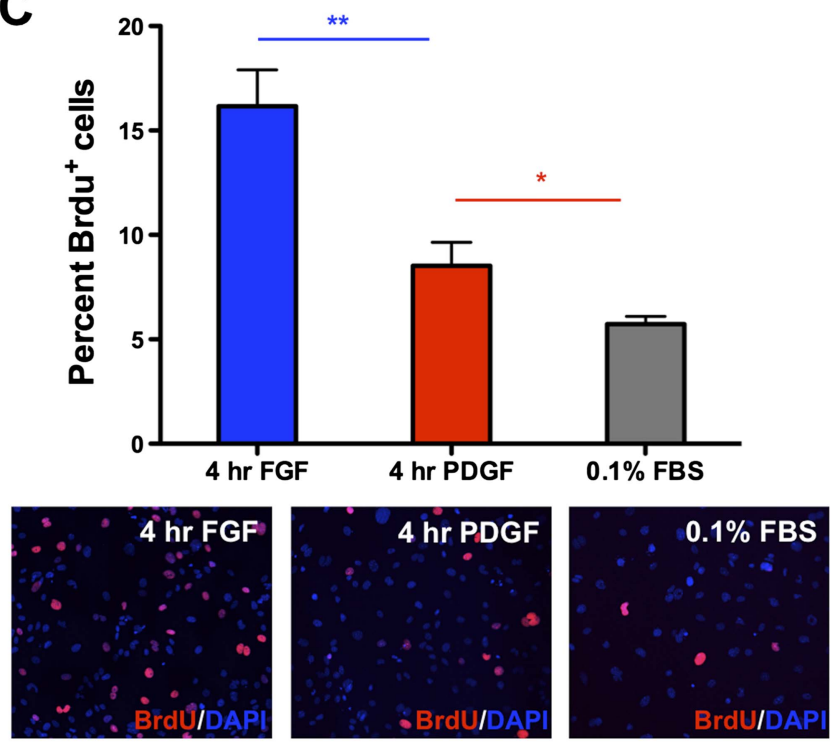

D
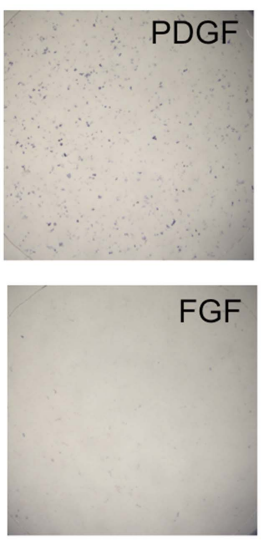
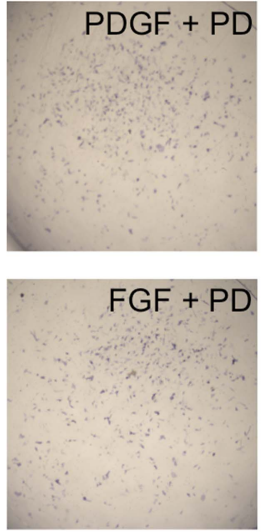

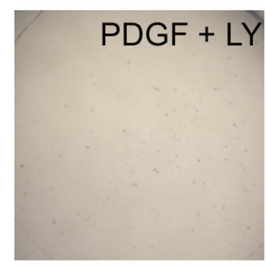

$F G F+L Y$
B
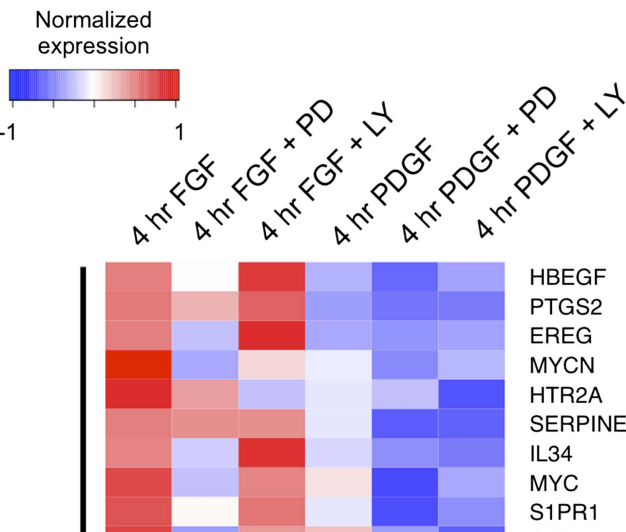

Figure 5. Distinct cellular outcomes are specified in response to PDGF and FGF signaling. (A) Gene ontology analysis of genes DE between the 4-hr FGF and 4-hr PDGF conditions shows enrichment for regulators of cell proliferation in genes upregulated by FGF treatment. In contrast, genes implicated in skeletal differentiation are overrepresented in the genes more highly expressed following PDGF treatment (B) Genes associated with cell proliferation are Figure 5. continued on next page 
Figure 5. Continued

strongly upregulated by FGF treatment, while genes associated with cell differentiation are increased following PDGF stimulation. In addition, MEK inhibition represses many proliferation genes but induces differentiation genes, while PI3K inhibition has the opposite effect. Genes ordered by decreasing ratio of FGF:PDGF expression. (C) FGF induces a significantly more robust proliferation response than PDGF in MEPMs, although PDGF does promote a modest response compared to starved cells (0.1\% FBS). Quantification plotted as mean $\pm S E M, n=3$. Two tailed, unpaired t-test: ${ }^{*} p<0.05$; ${ }^{* *} p<0.005$ (D) PDGF, but not FGF, treatment promotes alkaline phosphatase (AP) (osteoblast marker) positive cells. Furthermore, PD treatment drives AP staining, while LY treatment abolishes AP staining independent of growth factor stimulation. AP staining performed $8 \mathrm{hr}$ following ligand treatment. DOI: 10.7554/eLife.07186.010

The following figure supplement is available for figure 5 :

Figure supplement 1. PDGF-mediated differentiation responses exhibit a preference for PI3K signaling, while FGF-mediated effects on proliferation show greater MEK/ERK dependence. DOI: 10.7554/eLife.07186.011

(JNK-specific), and Dusp10 (JNK-specific) in the E13.5 palate by in situ hybridization (Figure 6-figure supplement 1A). Although Dusp1 and Dusp10 are primarily epithelial, Fgfr1 and Dusp6 are coexpressed in the anterior palatal mesenchyme, consistent with previous work implicating ERK in proliferation within this region (Bush and Soriano, 2010). Similarly, immunohistochemistry (IHC) revealed $\mathrm{pERK}$ is scattered in the anterior palatal mesenchyme, with some epithelial staining (Figure 6-figure supplement 1B). However, cell proliferation along the anterior-posterior axis is relatively uniform in the E13.5 palate (Bush and Jiang, 2012), complicating assignment of the observed expression patterns to a spatially restricted proliferation program. We next explored the relationship between PI3K signaling and osteoblast differentiation. Whole mount IHC for pAkt at E13.5 demonstrates expression restricted to the developing upper lip and middle to posterior palate, overlapping with AP in these structures (Figure 6A). We further assayed the pattern of Runx2 expression (Figure 6B) in comparison to Pdgfra, Id1, and Id3 in the E13.5 palate (Figure 6B'), finding shared domains of expression and exclusion in the palate. Taken together, this correlation between PI3K activity, the Id genes, and regions of osteoblast differentiation supports the existence of a spatially restricted PDGF-PI3K-differentiation axis.

In MEPMs, FGF drives proliferation and represses differentiation. One prediction from this observation is that Fgfr 1 mutants would have decreased repression of this program, and consequently, increased differentiation in the midface. We, therefore, performed AP staining on neural crest conditional Fgfr1 mutants (Wnt1-Cre; Fgfr ${ }^{f / f}$ ) at E14.5 to investigate defects in osteoblast differentiation. We observed an increased domain of AP in the maxillary region of Fgfr1 conditional mutants compared to heterozygous controls (Figure 6C). Collectively, these results suggest the FGF repressed, PI3K-dependent differentiation program identified in MEPMs is conserved in vivo.

\section{Discussion}

Our studies show that PDGF and FGF signaling in MEPMs regulate different gene expression programs and phenotypic outputs, with PDGF mainly promoting cell differentiation through PI3K and FGF favoring cell proliferation through ERK. Although the initial wave of gene expression shows high overlap, FGF elicits a quantitatively stronger response in terms of both signal magnitude and duration, which is reinforced by a positive signal from the delayed transcriptional wave in response to FGF but not PDGF. Furthermore, FGF-responsive genes are predominantly ERK dependent, while PDGF targets exhibit greater PI3K dependence genome-wide, relationships mimicked at the level of cellular outcome. Finally, we observed correlation between PDGF-PI3K signaling and osteoblast differentiation at E13.5 as well as increased AP staining in Fgfr1 mutants, indicating the differentiation circuit repressed by FGF signaling in MEPMs is functional in vivo.

The architecture of the transcriptional response to RTK signaling has been well described as three stereotypic waves: an IEG response, a delayed wave providing feedback control, and a late wave determining cellular outcome (Amit et al., 2007; Avraham and Yarden, 2011). In MEPMs, the magnitude and duration of the IEG wave is much stronger in response to FGF compared to PDGF, suggesting one level of specificity may be achieved through quantitative differences in IEG induction. Indeed, transient and sustained pERK induction, resulting in distinct magnitudes of Fos mRNA expression, drive binary responses in c-Fos abundance and activity at the protein level (Murphy et al., 2002; Nakakuki et al., 2010), delineating one mechanism through which quantitative differences lead to 
A

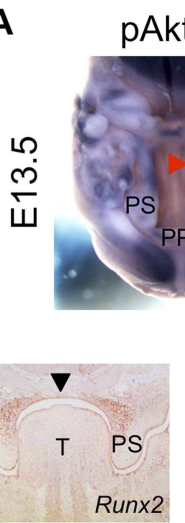

Akt

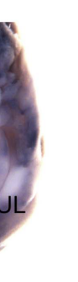

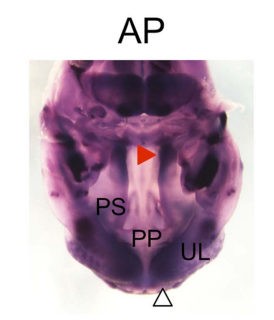

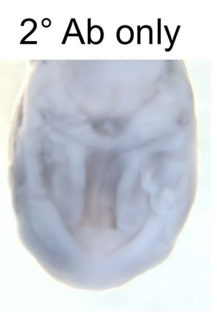

B'

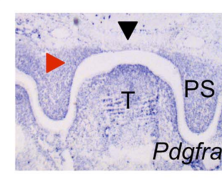

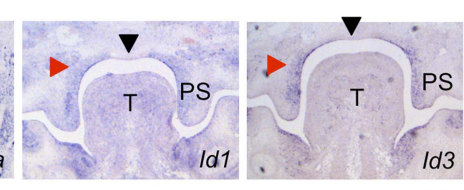

Id 3
C $\quad$ E14.5

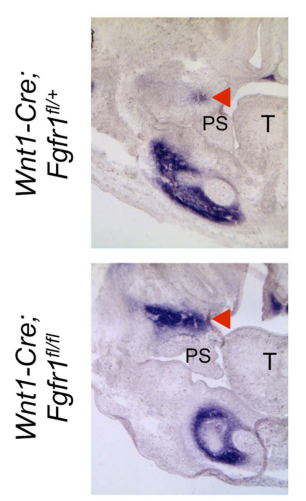

D

Superinduction:
Fos, Fosb, Junb,

Fos, Fosb, Junb
Dusp4, Dusp6

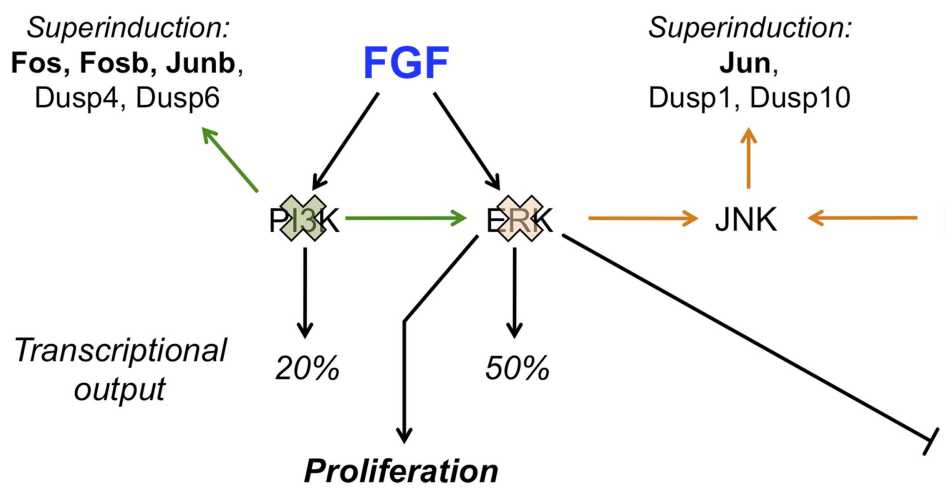

Superinduction:

Dusp1, Dusp10

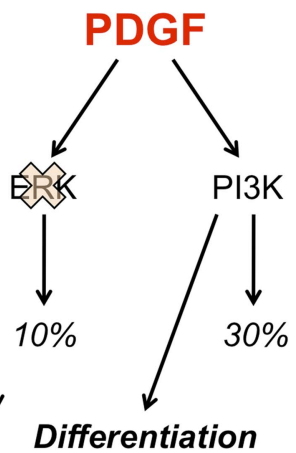

Figure 6. In vivo correlation and perturbation of the RTK-mediated differentiation program during mouse craniofacial development. (A) At E13.5, pAkt and AP domains co-localize in the middle to posterior secondary palate (red arrowhead) as well as in the developing upper lip (open arrowhead). (B) Domain of Runx2 (osteoblast marker) expression overlaps with ( $\left.\mathbf{B}^{\prime}\right)$ Pdgfra, $l d 1$, and $I d 3$ expression in the middle to posterior palate (red arrowhead), with expression excluded along the midline (black arrowhead). (C) Frontal sections from neural crest conditional Fgfr1 mutants (Wnt1-Cre; Fgfr $1^{f / f 1}$ ) exhibit increased AP staining in the developing midface at E14.5, supporting an in vivo role for FGF-mediated repression of osteoblast differentiation (red arrowhead) $(n=3)$. (D) FGF and PDGF signaling use different signaling pathways to instruct divergent cellular outcomes. FGF drives cell proliferation and represses cell differentiation in an ERK-dependent manner, consistent with a greater percentage of the FGF target genes being MEK/ERK dependent (50\%) than PI3K dependent (20\%). In contrast, PDGF promotes cell differentiation in a PI3K-dependent manner, and PDGF target genes show greater PI3K dependence (30\%) than MEK/ERK dependence (10\%). Furthermore, inhibition of PI3K signaling leads to an FGF specific induction of pERK (green) and consequently increased transcription of ERK targets such as Fos, Fosb, and Junb. On the other hand, MEK/ERK inhibition leads to pJNK induction (orange) and transcription of Jun, indicating multiple crosstalk mechanisms across different intracellular pathways in response to RTK activation. PP: primary palate; PS: palatal shelf; T: tongue; UL: upper lip.

DOI: 10.7554/eLife.07186.012

The following figure supplement is available for figure 6:

Figure supplement 1. Patterns of gene expression and pERK activity in the E13.5 palate. DOI: 10.7554/eLife.07186.013

a thresholded 'ON-OFF' response in downstream transcriptional activity. Thus, in addition to the observed PDGF-PI3K and FGF-ERK relationships, distinct patterns of IEG transcription factor expression may also contribute to the divergent gene expression profiles at later time points.

While inhibition of the delayed transcriptional response prolongs the PDGF-pERK wave, the opposite effect is observed on FGF-pERK induction. This result is consistent with the diversity of feedback and feedforward regulation on the RTK response (Avraham and Yarden, 2011; Volinsky and Kholodenko, 2013). In addition to transcriptional feedback, many other mechanisms, including ligand identity and receptor endocytosis (Francavilla et al., 2013), contribute toward 
specifying the signaling response to RTK activation. The complexity of this regulation underscores the intricate balance between positive and negative control systems, and it will be critical to determine how these regulatory mechanisms interact to produce the desired developmental outcomes.

Given the pleiotropic roles of RTKs, a central question involves how a common set of signal transduction modules specifies distinct outcomes. Our study supports a model in which differential intracellular pathway activation is responsible for the distinct transcriptional responses and cellular processes mediated by RTK signaling in MEPMs (Figure 6D). Pathway activation downstream of a single RTK can be affected by many parameters, such as receptor expression level (Traverse et alo, 1994; Tallquist et alo, 2003), suggesting that even a single receptor can regulate multiple downstream outputs and transcriptional programs across different contexts. The role of quantitative differences in the pERK response leading to distinct cellular outcomes is well characterized in PC12 neurons (Marshall, 1995), and the transcriptional response to the proliferative program mediated by sustained pERK activation in 3 T3 fibroblasts has been reported (Yamamoto et al., 2006). Building on these studies, the MEPM RNA-seq data provide insight into the differences in gene expression following both transient and sustained pERK induction as well as qualitatively specific target genes downstream of MEK/ERK and PI3K signaling, with the added advantage of profiling these responses within a system requiring these pathways for normal development. It is important to note that mRNA levels alone (as measured by RNA-seq) are not the only level of specificity in the transcriptional response, for example, differential cofactor recruitment can also specify distinct gene expression programs in response to FGF and PDGF signaling (Vasudevan and Soriano, 2014). Further work is necessary to identify the precise mechanisms regulating the activity of individual RTK target genes.

Although we focused on MEK/ERK and PI3K signaling based on their reported roles downstream of PDGF and FGF during development (Klinghoffer et alı, 2002; Corson et al., 2003), many other intracellular pathways are activated by RTKs, such as JNK, p38, Src, PLC $\gamma$, and PKC (Lemmon and Schlessinger, 2010). As previously reported in other contexts (Bhalla et al., 2002; Santos et al., 2007), we found the FGF-pERK induction was dependent on PKC activity, and we further identified crosstalk in the presence of pathway inhibition between ERK, PI3K, and JNK. In addition, a subset of genes (34\% FGF, $43 \%$ PDGF) are not affected by MEK/ERK or PI3K inhibition, suggesting they either lie downstream of other intracellular pathways or require inhibition of both ERK and PI3K. Thus, while our studies demonstrate PI3K is necessary for differentiation and ERK is necessary for proliferation in MEPMs, the high connectivity across intracellular pathways suggests no single pathway in isolation is sufficient to drive transcriptional responses and cellular outcomes in their entirety. Rather, integrated output from multiple effector pathways likely contributes to the ultimate cellular outcome, and instead of the existence of linear PDGF-PI3K-differentiation and FGF-ERK-proliferation axes, we favor a model in which multiple signaling events converge on PI3K to promote differentiation and ERK to drive proliferation in the midface.

The finding that effector inhibition increased the induction of many RTK target genes was surprising given their presumed primary function as positive effectors of signaling. The 'superinduction' of these transcriptional responses was due at least in part to compensatory activation of other pathways: inhibition of pERK downstream of both RTKs resulted in increased pJNK induction while inhibition of PI3K downstream of FGF, but not PDGF, resulted in increased pERK activation. This observation has implications for both genetic studies in mice and any system in which effector inhibition is used as an experimental or therapeutic agent. First, allelic series experiments in which adaptor binding is abrogated in order to abolish specific effector cascades may result in induction of alternate signaling pathways, complicating assignment of developmental function to a single intracellular pathway. Indeed, mice harboring a PI3K binding site mutation at the PDGFR locus show increased SHP2 binding and altered pERK activation (Klinghoffer et al., 2002). Second, chemical inhibition of these effectors may prime activation of other pathways, facilitating alternate signaling mechanisms and cellular outcomes. Many examples of such crosstalk between ERK and PI3K (Mendoza et al., 2011; Sun and Bernards, 2014) as well as ERK and JNK (Lopez-Bergami et alı, 2007) have been described downstream of RTK signaling in cancer. The MEPM RNA-seq data provide a transcriptional signature for this crosstalk, offering an additional readout to measure activation of these pathways. However, it is important to caution that these responses likely vary based on cellular context, and although the core target genes and effector dependencies may be conserved, extrapolating this framework to other systems requires careful validation.

In comparing our gene expression studies to mouse craniofacial development in vivo, we found colocalization of pAkt and AP in the upper lip and middle to posterior palate at E13.5 as well as 
overlapping domains of Runx2 and Id gene expression, indicating the PDGF-PI3K axis identified in MEPMs is correlated with osteoblast differentiation in the midface. It is interesting to note that the distribution of Pdgfra mRNA expression compared to pAkt activity and AP staining is not strictly one-toone; we speculate other factors such as ligand distribution, availability of intracellular signaling proteins, and input from other pathways may contribute toward the spatially restricted domains of osteoblast differentiation. We further observed elevated AP expression in Fgfr1 conditional mutants at E14.5, consistent with FGF-mediated repression of osteoblast differentiation. Many functions have been ascribed to FGF signaling in skeletogenesis (Ornitz and Marie, 2002), and a combination of parameters is likely responsible for the multiplicity of observed roles, such as ligand identity (Francavilla et al., 2013) and cellular context. During palatogenesis, Fgfr1 mutants have been previously reported to exhibit proliferation defects and increased BMP (Bone Morphogenetic Protein) signaling (Wang et al., 2013), supporting the notion that FGF drives proliferation and represses differentiation in this system. In addition, the facial clefting phenotypes associated with neural crest conditional loss of RAF, MEK, or ERK (Newbern et alo, 2008) suggest that the FGF-ERK axis is functionally relevant in vivo.

One important point merits further discussion: Are PI3K and ERK induction interpreted the same independently of the stimulus driving effector activity? There is evidence to suggest this is indeed the case, as IGF (Insulin-like growth factor 1)-mediated PI3K/Akt activation is a key regulator of osteoblast differentiation in mesenchymal stem cells (Xian et al., 2012), consistent with the PI3K-mediated differentiation outcome in MEPMs. However, this interpretation is likely restricted in large part by cellular context, as MEK/ERK signaling functions as a positive regulator of differentiation in embryonic stem cells (Ying et al., 2008), in contrast to the role of MEK/ERK signaling in MEPMs. In skeletal differentiation, our findings of a MEK/ERK-mediated proliferation program and PI3K-mediated differentiation program are in line with previous work analyzing the role of these effectors (Mansukhani et al., 2005; Raucci et al., 2008; Miraoui and Marie, 2010). In addition, a FGF-PKCAP-1 signaling axis in calvarial osteoblasts has been reported (Miraoui et al., 2010), consistent with the MEPM data linking FGF to a robust AP-1 response and FGF-mediated pERK activation to PKC signaling. Delineating the hierarchy between these signals and pathways and determining the exact combinations sufficient to specify particular outcomes will be key questions for future studies. The present work provides a roadmap of the gene expression profiles underlying these cellular behaviors and a transcriptomic view of how two different RTKs lead to distinct outcomes.

\section{Materials and methods}

\section{Mouse strains}

All animal experiments were approved by the Institutional Animal Care and Use Committee at the Icahn School of Medicine at Mount Sinai. Wild-type C57B/6 mice were used to generate E13.5 MEPMs for RNA-

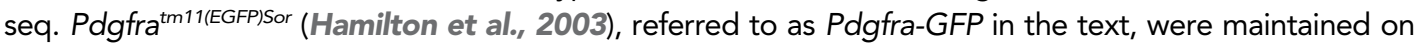
a C57BL/6 background, and FGFR1-CFP mice (to be described elsewhere), FGFR1 ${ }^{\text {tm5.15or }}$ mice (Hoch and Soriano, 2006), referred to as Fgfr $1^{f / f l}$ in the text, and $\mathrm{Tg}$ (Wnt1-Cre)11Rth mice (Danielian et al., 1998), referred to as Wnt1-Cre in the text, were all maintained on a 129S4 background.

\section{Tissue culture}

Primary MEPM cells were isolated from E13.5 secondary palatal shelves (day of plug: E0.5) as previously described (Fantauzzo and Soriano, 2014; Vasudevan and Soriano, 2014). Cells were grown in Dulbecco's modified Eagle's medium (GIBCO; Invitrogen, Carlsbad, CA) with 10\% fetal bovine serum (FBS; HyClone Laboratories, Logan, UT), $50 \mathrm{U} / \mathrm{mL}$ penicillin (GIBCO), $50 \mu \mathrm{g} / \mathrm{mL}$ streptomycin (GIBCO), and $2 \mathrm{mM}$ L-glutamine (GIBCO). Cells were split twice to passage 2 for all experiments included in this study, and serum starvation was conducted in same media as above but with $0.1 \%$ FBS instead of 10\% FBS. BrdU assays (Bush and Soriano, 2010; Vasudevan and Soriano, 2014) and AP staining (Wu et al., 2008) were performed as described previously.

\section{RNA-seq}

Passage 2 E13.5 MEPMs were serum starved overnight in $0.1 \% \mathrm{FBS}$ and then treated with either $30 \mathrm{ng} / \mathrm{mL}$ PDGFAA (R\&D Systems, Minneapolis, MN) or 50 ng/mL FGF1 (Peprotech, Rocky Hill, NJ) $+1 \mu \mathrm{g} / \mathrm{mL}$ heparin (Sigma-Aldrich, St. Louis, MO) for the desired duration. When pathway inhibitors were used, cells were pretreated with either $1 \mu \mathrm{M}$ PD325901 or $10 \mu \mathrm{M}$ LY294002 for 30 min prior to growth factor 
addition. MEPMs generated from independent litters were used for each set of replicates, and RNA was collected using RNeasy Mini Kit (Qiagen Inc., Valencia, CA) according to manufacturer's protocol before submission to the Mount Sinai Genomics Core (http://icahn.mssm.edu/departments-andinstitutes/genomics/about/resources/genomics-core-facility), where RNA was poly-A selected, libraries generated, and sequenced on Illumina HiSeq 2000. Between 25 and 40 million reads per sample were obtained and mapped to the mouse genome (version mm10) using TopHat (Kim et al., 2013). Genes were tested for differential expression by Cuffdiff and considered significant at $q<0.1$ (Trapnell et al., 2010). Data for untreated, 1-hr PDGF, and 1-hr FGF treated MEPMs are publicly available at the Gene Expression Omnibus (GEO), Accession GSE61755 (42). Data for $4 \mathrm{hr}$ PDGF, $4 \mathrm{hr}$ FGF, 1 hr PDGF + PD325901, 1 hr PDGF + LY294002, 4 hr PDGF + PD325901, 4 hr PDGF + LY294002, $1 \mathrm{hr} F G F+$ PD325901, 1 hr FGF + LY294002, 4 hr FGF + PD325901, and 4 hr FGF + LY294002 samples are publicly available at the GEO, Accession GSE66484.

\section{RT-qPCR}

E13.5 MEPMs were serum starved overnight in 0.1\% FBS and then treated with desired growth factors and/or inhibitors as for RNA-seq. Total RNA was collected with the RNeasy Mini Kit (Qiagen Inc.). First-strand CDNA was then synthesized using a ratio of 2:1 random primers: Oligo(dT) with SuperScript II RT (Invitrogen). qPCR was performed using a Bio-Rad iQ5 Multicolor Real-Time PCR Detection System and analyzed with iQ5 Optical System Software (version 2.0; Bio-Rad, Hercules, CA). Reactions were performed with PerfeCTa SYBR Green FastMix for iQ (Quanta Biosciences Inc., Gaithersburg, MD) using $10 \mu \mathrm{M}$ primers (Integrated DNA Technologies Inc., Coralville, IA). A list of qPCR primers used is available in Supplementary File 5. The following cycling protocol was used: step 1: $95^{\circ} \mathrm{C}$ for $3 \mathrm{~min}$; step 2: $95^{\circ} \mathrm{C}$ for $10 \mathrm{~s}$; step 3: $60^{\circ}$ for $40 \mathrm{~s}$; repeat to step $239 \times$ (total of 40 cycles), and a melting curve analysis was performed. In addition, PCR products were run on a $1.0 \%$ agarose gel to ensure correct amplicon size. $\beta 2 \mathrm{~m}$ was used as an endogenous control.

\section{Western blot}

MEPMs were serum starved overnight and treated as for RNA-seq and then washed $3 \times$ in ice-cold PBS (Phosphate-buffered saline) before being harvested in NP-40 lysis buffer (20 mM Tris-HCl pH 8, 150 $\mathrm{mM} \mathrm{NaCl}$, 10\%glycerol, 1\% Nonidet P-40, 2 mM EDTA (Ethylenediaminetetraacetic acid), 1× complete Mini protease inhibitor cocktail [Roche Applied Science, Indianapolis, IN], 1 mM PMSF (Phenylmethanesulfonylfluoride), $10 \mathrm{mM} \mathrm{NaF}, 1 \mathrm{mM} \mathrm{Na3VO4}$, and $25 \mathrm{mM} \beta$-glycerophosphate). Total cell lysates were sonicated briefly and then collected by centrifugation. Lysates were then resuspended in Laemmli buffer containing $10 \% \beta$-mercaptoethanol, heated at $95^{\circ} \mathrm{C}$ for $5 \mathrm{~min}$, and separated by SDS-polyacrylamide gel electrophoresis.

The following inhibitors were used: LY294002 (Sigma-Aldrich), PD325901 (Stemgent, Cambridge, MA), cycloheximide (Fisher Scientific, Waltham, MA), and Bim I (Santa Cruz Biotechnology, Dallas, TX).

The following antibodies were used: anti-phospho MAPK p42/p44 (9201; Cell Signaling Technologies, Danvers, MA; 1:1000), anti-pAkt (9271; Cell Signaling Technologies; 1:1000), and antipJNK (4671; Cell Signaling Technologies; $1: 1000)$. The anti- $\beta$ tubulin E7 antibody (1:1000) developed by M. Klymkowsky was obtained from the Developmental Studies Hybridoma Bank developed under the auspices of the NICHD and maintained by The University of lowa, Department of Biology, lowa City, IA.

\section{Immunofluorescence}

Immunofluorescence was performed as described previously (Vasudevan and Soriano, 2014). Briefly, cells were fixed in $4 \%$ formaldehyde at room temperature, blocked in $10 \%$ donkey serum, stained with primary antibody, and detected with secondary antibody, all for $1 \mathrm{hr}$ at room temperature. The following antibodies were used: Anti-Cleaved Caspase-3 (CST9661; Cell Signaling Technology; 1:400). The anti-BrdU (G3G4, 1:500) developed by S. Kaufman was obtained from the Developmental Studies Hybridoma Bank, created by the NICHD of the NIH and maintained at The University of lowa, Department of Biology, lowa City, IA.

\section{IHC}

IHC was performed as described previously (Fantauzzo and Soriano, 2014). For whole mounts, embryos were dissected onto ice-cold PBS, fixed overnight in 4:1 methanol:DMSO, cleared in 4:1:1 methanol:DMSO: $\mathrm{H}_{2} \mathrm{O}_{2}$, and stored in $100 \%$ methanol. For sections, embryos were fixed overnight in 
4\% paraformaldehyde (PFA) and embedded in paraffin. Staining was done with primary antibody overnight and a goat anti-rabbit IgG peroxidase-conjugated secondary antibody (Jackson ImmunoResearch Laboratories Inc., West Grove, PA). Detection was performed using the Vector Laboratories SK-4100 kit (Vector Laboratories Inc., Burlingame, CA).

\section{In situ hybridization}

Embryos were dissected in ice-cold PBS and fixed overnight in 4\% PFA and embedded in paraffin or optimal cutting temperature compound for sectioning. In situ hybridization was performed according to standard protocols (He and Soriano, 2013; Vasudevan and Soriano, 2014). A list of probe sequences is provided in Supplementary File 5.

\section{Data analysis}

Generation of heatmaps, PCA, and other analysis were performed through custom code in R. PCA was done using the 'prcomp' function on centered, median normalized data, and correlation matrix was constructed using the 'cor' function on $\log _{2}(F P K M+1)$ transformed data. Hierarchical clustering and heatmaps were generated using the 'heatmap.2' and 'hclust' functions. Pearson's correlation was used in the correlation matrix, and Euclidean distance was used as a distance metric for hierarchical clustering. PPI networks were constructed using the Expression2Kinase software (Chen et al., 2012) based on an updated version of Genes2Networks (Berger et al., 2007), only direct connections (path length $=1$ ) were considered, and all published PPI databases except predicted PPIs were included. Networks were visualized and formatted in yEd (www.yworks.com). For Gene ontology analysis (Huang et alo, 2009), only those terms at a significance threshold of $p<0.001$ were included. Redundant GO terms comprising an identical or fully shared subset of genes were removed. A full list of GO results is provided in Supplementary File 4.

\section{Acknowledgements}

We are very grateful to Avi Ma'ayan for advice and thoughtful comments on data analysis and visualization. We thank Tony Chen, Aryel Heller, and Anne Levine for excellent technical support; Omar Jabado, Yumi Kasai, and Milind Mahajan for advice and discussions on RNA-seq experiments performed at the Mount Sinai Genomics Core; and members of our laboratory, Andy Chess and Stu Aaronson for helpful discussion and critical comments on this manuscript.

\section{Additional information}

Funding

\begin{tabular}{lll} 
Funder & Grant reference & Author \\
\hline National Institutes of Health & R01DE022363 & Philippe Soriano \\
\hline National Institutes of Health & R01DE022778 & Philippe Soriano \\
\hline National Institutes of Health & $\begin{array}{l}\text { NRSA Individual Predoctoral } \\
\text { Fellowship F31DE023456 }\end{array}$ & $\begin{array}{l}\text { Harish N } \\
\text { Vasudevan }\end{array}$ \\
\hline National Institutes of Health & K99DE024617 & Fenglei He \\
\hline
\end{tabular}

The funder had no role in study design, data collection and interpretation, or the decision to submit the work for publication.

Author contributions

HNV, PM, Conception and design, Acquisition of data, Analysis and interpretation of data, Drafting or revising the article; $\mathrm{FH}$, Acquisition of data, Analysis and interpretation of data, Drafting or revising the article; PS, Conception and design, Analysis and interpretation of data, Drafting or revising the article

Ethics

Animal experimentation: This study was performed in strict accordance with the recommendations in the Guide for the Care and Use of Laboratory Animals of the National Institutes of Health. All of the animals were handled according to approved Institutional Animal Care and Use Committee (IACUC) protocols (\#11-00243) of Icahn School of Medicine at Mt. Sinai. 


\section{Additional files}

Supplementary files

- Supplementary file 1. FPKM values for all genes across all sequenced conditions.

DOI: 10.7554/eLife.07186.014

- Supplementary file 2. Expression for all differentially expressed genes (Cuffdiff $q<0.1$ ).

DOI: 10.7554/eLife.07186.015

- Supplementary file 3. Inhibitor dependence for RTK target genes.

DOI: 10.7554/eLife.07186.016

- Supplementary file 4. Compiled gene ontology analysis.

DOI: 10.7554/eLife.07186.017

- Supplementary file 5. PCR primers and in situ hybridization probes.

DOI: 10.7554/eLife.07186.018

Major datasets

The following datasets were generated:

\begin{tabular}{lllll}
\hline Author(s) & Year & Dataset title & $\begin{array}{l}\text { Dataset ID } \\
\text { and/or URL }\end{array}$ & $\begin{array}{l}\text { Database, license, and } \\
\text { accessibility information }\end{array}$ \\
\hline $\begin{array}{l}\text { Vasudevan } \\
\text { HN }\end{array}$ & $2014 \begin{array}{l}\text { PDGF and FGF treatment in } \\
\text { E13.5 MEPMs }\end{array}$ & $\begin{array}{l}\text { http://www.ncbi.nlm.nih.gov/ } \\
\text { geo/query/acc.cgi? } \\
\text { acc=GSE61755 }\end{array}$ & $\begin{array}{l}\text { Publicly available at NCBI } \\
\text { Gene Expression Omnibus } \\
\text { (GSE61755). }\end{array}$ \\
$\begin{array}{llll}\text { Vasudevan } \\
\text { HN }\end{array}$ & $2015 \begin{array}{l}\text { PDGF and FGF treatment in } \\
\text { E13.5 MEPMs II }\end{array}$ & $\begin{array}{l}\text { http://www.ncbi.nlm.nih.gov/ } \\
\text { geo/query/acc.cgi? } \\
\text { acc=GSE66484 }\end{array}$ & $\begin{array}{l}\text { Publicly available at NCBI } \\
\text { Gene Expression Omnibus } \\
\text { (GSE66484). }\end{array}$
\end{tabular}

\section{References}

Amit I, Citri A, Shay T, Lu Y, Katz M, Zhang F, Tarcic G, Siwak D, Lahad J, Jacob-Hirsch J, Amariglio N, Vaisman N, Segal E, Rechavi G, Alon U, Mills GB, Domany E, Yarden Y. 2007. A module of negative feedback regulators defines growth factor signaling. Nature Genetics 39:503-512. doi: 10.1038/ng1987.

Avraham R, Yarden Y. 2011. Feedback regulation of EGFR signalling: decision making by early and delayed loops. Nature Reviews. Molecular Cell Biology 12:104-117. doi: 10.1038/nrm3048.

Bentires-Alj M, Kontaridis MI, Neel BG. 2006. Stops along the RAS pathway in human genetic disease. Nature Medicine 12:283-285. doi: 10.1038/nm0306-283.

Berger SI, Posner JM, Ma'ayan A. 2007. Genes2Networks: connecting lists of gene symbols using mammalian protein interactions databases. BMC Bioinformatics 8:372. doi: 10.1186/1471-2105-8-372.

Bhalla US, Ram PT, lyengar R. 2002. MAP kinase phosphatase as a locus of flexibility in a mitogen-activated protein kinase signaling network. Science 297:1018-1023. doi: 10.1126/science.1068873.

Bush JO, Jiang R. 2012. Palatogenesis: morphogenetic and molecular mechanisms of secondary palate development. Development 139:828-828. doi: 10.1242/dev.079152.

Bush JO, Soriano P. 2010. Ephrin-B1 forward signaling regulates craniofacial morphogenesis by controlling cell proliferation across Eph-ephrin boundaries. Genes \& Development 24:2068-2080. doi: 10.1101/gad. 1963210.

Caloca MJ, Zugaza JL, Matallanas D, Crespo P, Bustelo XR. 2003. Vav mediates Ras stimulation by direct activation of the GDP/GTP exchange factor Ras GRP1. EMBO Journal 22:3326-3336. doi: 10.1093/emboj/ codg316.

Chen EY, Tan CM, Kou Y, Duan Q, Wang Z, Meirelles GV, Clark NR, Ma'ayan A. 2013. Enrichr: interactive and collaborative HTML5 gene list enrichment analysis tool. BMC Bioinformatics 14:128. doi: 10.1186/1471. 2105-14-128.

Chen EY, Xu H, Gordonov S, Lim MP, Perkins MH, Ma'ayan A. 2012. Expression2Kinases: mRNA profiling linked to multiple upstream regulatory layers. Bioinformatics 28:105-111. doi: 10.1093/bioinformatics/btr625.

Choi SJ, Marazita ML, Hart PS, Sulima PP, Field LL, McHenry TG, Govil M, Cooper ME, Letra A, Menezes R, Narayanan S, Mansilla MA, Granjeiro JM, Vieira AR, Lidral AC, Murray JC, Hart TC. 2009. The PDGF-C regulatory region SNP rs28999109 decreases promoter transcriptional activity and is associated with CL/P. European Journal of Human Genetics: EJHG 17:774-784. doi: 10.1038/ejhg.2008.245.

Cochran BH, Reffel AC, Stiles CD. 1983. Molecular cloning of gene sequences regulated by platelet-derived growth factor. Cell 33:939-947. doi: 10.1016/0092-8674(83)90037-5.

Corson LB, Yamanaka Y, Lai KM, Rossant J. 2003. Spatial and temporal patterns of ERK signaling during mouse embryogenesis. Development 130:4527-4537. doi: 10.1242/dev.00669.

Danielian PS, Muccino D, Rowitch DH, Michael SK, McMahon AP. 1998. Modification of gene activity in mouse embryos in utero by a tamoxifen-inducible form of Cre recombinase. Current biology 8:1323-1326. doi: 10.1016/ S0960-9822(07)00562-3. 
Ding H, Wu X, Boström H, Kim I, Wong N, Tsoi B, O'Rourke M, Koh GY, Soriano P, Betsholtz C, Hart TC, Marazita ML, Field LL, Tam PP, Nagy A. 2004. A specific requirement for PDGF-C in palate formation and PDGFR-alpha signaling. Nature Genetics 36:1111-1116. doi: 10.1038/ng1415.

Fambrough D, McClure K, Kazlauskas A, Lander ES. 1999. Diverse signaling pathways activated by growth factor receptors induce broadly overlapping, rather than independent, sets of genes. Cell 97:727-741. doi: 10.1016/ S0092-8674(00)80785-0.

Fantauzzo KA, Soriano P. 2014. PI3K-mediated PDGFR $\alpha$ signaling regulates survival and proliferation in skeletal development through p53-dependent intracellular pathways. Genes \& Development 28:1005-1017. doi: 10 1101/gad.238709.114.

Fantauzzo KA, Soriano P. 2015. Receptor tyrosine kinase signaling: regulating neural crest development one phosphate at a time. Current Topics in Developmental Biology 111:135-182. doi: 10.1016/bs.ctdb.2014.11.005.

Francavilla C, Rigbolt KT, Emdal KB, Carraro G, Vernet E, Bekker-Jensen DB, Streicher W, Wikström M, Sundström M, Bellusci S, Cavallaro U, Blagoev B, Olsen JV. 2013. Functional proteomics defines the molecular switch underlying FGF receptor trafficking and cellular outputs. Molecular Cell 51:707-722. doi: 10.1016/j.molcel.2013.08.002.

Gotoh T, Cai D, Tian X, Feig L, Lerner A. 2000. p130(Cas) regulates the activity of AND-34, a novel Ral, Rap1, and R-Ras guanine nucleotide exchange factor. Journal of Biological Chemistry 275:30118-30123. doi: 10.1074/jbc. M003074200.

Hamilton TG, Klinghoffer RA, Corrin PD, Soriano P. 2003. Evolutionary divergence of platelet-derived growth factor alpha receptor signaling mechanisms. Molecular and Cellular Biology 23:4013-4025. doi: 10.1128/MCB. 23.11.4013-4025.2003.

He F, Soriano P. 2013. A critical role for PDGFR signaling in medial nasal process development. Plos Genetics 9: e1003851. doi: 10.1371/journal.pgen.1003851.

Hoch RV, Soriano P. 2006. Context-specific requirements for Fgfr1 signaling through Frs2 and Frs3 during mouse development. Development 133:663-673. doi: 10.1242/dev.02242.

Huang DW, Sherman BT, Lempicki RA. 2009. Systematic and integrative analysis of large gene lists using DAVID bioinformatics resources. Nature Protocols 4:44-57. doi: 10.1038/nprot.2008.211.

Hunter T. 2000. Signaling-2000 and beyond. Cell 100:113-127. doi: 10.1016/S0092-8674(00)81688-8.

Iwata J, Hacia JG, Suzuki A, Sanchez-Lara PA, Urata M, Chai Y. 2012. Modulation of noncanonical TGF- $\beta$ signaling prevents cleft palate in Tgfbr2 mutant mice. The Journal of Clinical Investigation 122:873-885. doi: 10.1172/ JCl61498.

Kee Y, Bronner-Fraser M. 2005. To proliferate or to die: role of Id3 in cell cycle progression and survival of neural crest progenitors. Genes \& Development 19:744-755. doi: 10.1101/gad.1257405.

Kim D, Pertea G, Trapnell C, Pimentel H, Kelley R, Salzberg SL. 2013. TopHat2: accurate alignment of transcriptomes in the presence of insertions, deletions and gene fusions. Genome Biology 14:R36. doi: 10.1186/ gb-2013-14-4-r36.

Kiss-Toth E, Bagstaff SM, Sung HY, Jozsa V, Dempsey C, Caunt JC, Oxley KM, Wyllie DH, Polgar T, Harte M, O'Neill LA, Qwarnstrom EE, Dower SK. 2004. Human tribbles, a protein family controlling mitogenactivated protein kinase cascades. Journal of Biological Chemistry 279:42703-42708. doi: 10.1074/jbc. M407732200.

Klinghoffer RA, Hamilton TG, Hoch R, Soriano P. 2002. An allelic series at the PDGF $\alpha$ R locus indicates unequal contributions of distinct signaling pathways during development. Developmental Cell 2:103-113. doi: 10.1016/ S1534-5807(01)00103-4.

Lanner F, Rossant J. 2010. The role of FGF/Erk signaling in pluripotent cells. Development 137:3351-3360. doi: 10. 1242/dev.050146.

Lau LF, Nathans D. 1987. Expression of a set of growth-related immediate early genes in BALB/c 3T3 cells: coordinate regulation with c-fos or c-myc. Proceedings of the National Academy of Sciences of USA 84: 1182-1186. doi: 10.1073/pnas.84.5.1182.

Lemmon MA, Schlessinger J. 2010. Cell signaling by receptor tyrosine kinases. Cell 141:1117-1134. doi: 10.1016/j. cell.2010.06.011.

Li C, Scott DA, Hatch E, Tian X, Mansour SL. 2007. Dusp6 (Mkp3) is a negative feedback regulator of FGFstimulated ERK signaling during mouse development. Development 134:167-176. doi: 10.1242/dev.02701.

Li L, Sun L, Gao F, Jiang J, Yang Y, Li C, Gu J, Wei Z, Yang A, Lu R, Ma Y, Tang F, Won Kwon W, Zhao Y, Li J, Jin Y. 2010. Stk40 links the pluripotency factor Oct4 to the Erk/MAPK pathway and controls extraembryonic endoderm differentiation. Proceedings of the National Academy of Sciences of USA 107:1402-1407. doi: 10.1073/pnas. 0905657107.

Lopez-Bergami P, Huang C, Goydos JS, Yip D, Bar-Eli M, Herlyn M, Smalley KS, Mahale A, Eroshkin A, Aaronson S, Ronai Z. 2007. Rewired ERK-JNK signaling pathways in melanoma. Cancer Cell 11:447-460. doi: 10.1016/j.ccr. 2007.03.009.

Maeda Y, Tsuji K, Nifuji A, Noda M. 2004. Inhibitory helix-loop-helix transcription factors Id1/ld3 promote bone formation in vivo. Journal of Cellular Biochemistry 93:337-344. doi: 10.1002/jcb.20154.

Mansukhani A, Ambrosetti D, Holmes G, Cornivelli L, Basilico C. 2005. Sox2 induction by FGF and FGFR2 activating mutations inhibits Wnt signaling and osteoblast differentiation. The Journal of Cell Biology 168: 1065-1076. doi: 10.1083/jcb.200409182.

Marshall CJ. 1995. Specificity of receptor tyrosine kinase signaling: transient versus sustained extracellular signalregulated kinase activation. Cell 2:179-185. doi: 10.1016/0092-8674(95)90401-8.

Mendoza MC, Er EE, Blenis J. 2011. The Ras-ERK and PI3K-mTOR pathways: cross-talk and compensation. Trends in Biochemical Sciences 36:320-328. doi: 10.1016/j.tibs.2011.03.006. 
Miraoui H, Ringe J, Häupl T, Marie PJ. 2010. Increased EFG- and PDGFalpha-receptor signaling by mutant FGFreceptor 2 contributes to osteoblast dysfunction in Apert craniosynostosis. Human Molecular Genetics 19: 1678-1689. doi: 10.1093/hmg/ddq045.

Miraoui H, Marie PJ. 2010. Fibroblast growth factor receptor signaling crosstalk in skeletogenesis. Science Signaling 3:re9. doi: 10.1126/scisignal.3146re9.

Murphy LO, Smith S, Chen RH, Fingar DC, Blenis J. 2002. Molecular interpretation of ERK signal duration by immediate early gene products. Nature Cell Biology 4:556-564. doi: 10.1038/ncb822.

Nakakuki T, Birtwistle MR, Saeki Y, Yumoto N, Ide K, Nagashima T, Brusch L, Ogunnaike BA, Okada-Hatakeyama M, Kholodenko BN. 2010. Ligand-specific c-fos expression emerges from the spatiotemporal control of ErbB network dynamics. Cell 141:884-896. doi: 10.1016/j.cell.2010.03.054.

Newbern J, Zhong J, Wickramasinghe RS, Li X, Wu Y, Samuels I, Cherosky N, Karlo JC, O'Loughlin B, Wikenheiser J, Gargesha M, Doughman YQ, Charron J, Ginty DD, Watanabe M, Saitta SC, Snider WD, Landreth GE. 2008. Mouse and human phenotypes indicate a critical conserved role for ERK2 signaling in neural crest development. Proceedings of the National Academy of Sciences of USA 105:17115-17120. doi: 10.1073/pnas.0805239105.

Ornitz D, Marie P. 2002. FGF signaling pathways in endochondral and intramembranous bone development and human genetic disease. Genes \& Development 16:1446-1465. doi: 10.1101/gad.990702.

Owens DM, Keyse SM. 2007. Differential regulation of MAP kinase signalling by dual-specificity protein phosphatases. Oncogene 26:3203-3213. doi: 10.1038/sj.onc.1210412.

Pawson T. 2004. Specificity in signal transduction: from phosphotyrosine-SH2 domain interactions to complex cellular systems. Cell 116:191-203. doi: 10.1016/S0092-8674(03)01077-8.

Rattanasopha S, Tongkobpetch S, Srichomthong C, Siriwan P, Suphapeetiporn K, Shotelersuk V. 2012. PDGFRa mutations in humans with isolated cleft palate. European Journal of Human Genetics: EJHG 20:1058-1062. doi: 10.1038/ejhg.2012.55.

Raucci A, Bellosta P, Grassi R, Basilico C, Mansukhani A. 2008. Osteoblast proliferation or differentiation is regulated by relative strengths of opposing signaling pathways. Journal of Cellular Physiology 215:442-451. doi: 10.1002/jcp.21323.

Santos SD, Verveer PJ, Bastiaens PI. 2007. Growth factor-induced MAPK network topology shapes Erk response determining PC-12 cell fate. Nature Cell Biology 9:324-330. doi: 10.1038/ncb1543.

Schmahl J, Raymond CS, Soriano P. 2007. PDGF signaling specificity is mediated through multiple immediate early genes. Nature Genetics 39:52-60. doi: 10.1038/ng1922.

Simon MA. 2000. Receptor tyrosine kinases: specific outcomes from general signals. Cell 103:13-15. doi: 10.1016/ S0092-8674(00)00100-8.

Soriano P. 1997. The PDGF $\alpha$ receptor is required for neural crest cell development and for normal patterning of the somites. Development 124:2691-2700.

Sun C, Bernards R. 2014. Feedback and redundancy in receptor tyrosine kinase signaling: relevance to cancer therapies. Trends in Biochemical Sciences 39:465-474. doi: 10.1016/j.tibs.2014.08.010.

Tallquist MD, French WJ, Soriano P. 2003. Additive effects of PDGF receptor $\beta$ signaling pathways in vascular smooth muscle cell development. Plos Biology 1:288-299. doi: 10.1371/journal.pbio.0000052.

Tallquist MD, Soriano P. 2003. Cell autonomous requirement for PDGFRalpha in populations of cranial and cardiac neural crest cells. Development 130:507-518. doi: 10.1242/dev.00241.

Trapnell C, Williams BA, Pertea G, Mortazavi A, Kwan G, van Baren MJ, Salzberg SL, Wold BJ, Pachter L. 2010. Transcript assembly and quantification by RNA-Seq reveals unannotated transcripts and isoform switching during cell differentiation. Nature Biotechnology 28:511-515. doi: 10.1038/nbt.1621.

Traverse S, Seedorf K, Paterson H, Marshall CJ, Cohen P, Ullrich A. 1994. EGF triggers neuronal differentiation of PC12 cells that overexpress the EGF receptor. Current biology 4:694-701. doi: 10.1016/S0960-9822(00) 00154-8.

Trokovic N, Trokovic R, Mai P, Partanen J. 2003. Fgfr1 regulates patterning of the pharyngeal region. Genes \& Development 17:141-153. doi: 10.1101/gad.250703.

Vasudevan HN, Soriano P. 2014. Article SRF regulates craniofacial development through selective recruitment of MRTF cofactors by PDGF signaling. Developmental Cell 31:332-344. doi: 10.1016/j. devcel. 2014.10.005.

Verzi MP, Agarwal P, Brown C, McCulley DJ, Schwarz JJ, Black BL. 2007. The transcription factor MEF2C is required for craniofacial development. Developmental Cell 12:645-652. doi: 10.1016/j.devcel. 2007.03.007.

Volinsky N, Kholodenko BN. 2013. Complexity of receptor tyrosine kinase signal processing. Cold Spring Harbor Perspectives in Biology 5:a009043. doi: 10.1101/cshperspect.a009043.

Wang C, Chang JY, Yang C, Huang Y, Liu J, You P, McKeehan WL, Wang F, Li X. 2013. Type 1 fibroblast growth factor receptor in cranial neural crest cell-derived mesenchyme is required for palatogenesis. The Journal of Biological Chemistry 288:22174-22183. doi: 10.1074/jbc.M113.463620.

Won M, Park KA, Byun HS, Kim YR, Choi BL, Hong JH, Park J, Seok JH, Lee YH, Cho CH, Song IS, Kim YK, Shen HM, Hur GM. 2009. Protein kinase SGK1 enhances MEK/ERK complex formation through the phosphorylation of ERK2: Implication for the positive regulatory role of SGK1 on the ERK function during liver regeneration. Journal of Hepatology 51:67-76. doi: 10.1016/j.jhep.2009.02.027.

Wu M, Li J, Engleka KA, Zhou B, Lu MM, Plotkin JB, Epstein JA. 2008. Persistent expression of Pax3 in the neural crest causes cleft palate and defective osteogenesis in mice. The Journal of Clinical Investigation 118:2076-2087. doi: $10.1172 / \mathrm{JCl} 33715$. 
Xian L, Wu X, Pang L, Lou M, Rosen CJ, Qiu T, Crane J, Frassica F, Zhang L, Rodriguez JP, Xiaofeng Jia, Shoshana Yakar, Shouhong Xuan, Argiris Efstratiadis, Mei Wan, Xu Cao. 2012. Matrix IGF-1 maintains bone mass by activation of mTOR in mesenchymal stem cells. Nature Medicine 18:1095-1101. doi: 10.1038/nm.2793.

Yamamoto T, Ebisuya M, Ashida F, Okamoto K, Yonehara S, Nishida E. 2006. Continuous ERK activation downregulates antiproliferative genes throughout $\mathrm{G} 1$ phase to allow cell-cycle progression. Current Biology 16: 1171-1182. doi: 10.1016/j.cub.2006.04.044.

Ying Q-L, Wray J, Nichols J, Batlle-Morera L, Doble B, Woodgett J, Cohen P, Smith A. 2008. The ground state of embryonic stem cell self-renewal. Nature 453:519-523. doi: 10.1038/nature06968. 